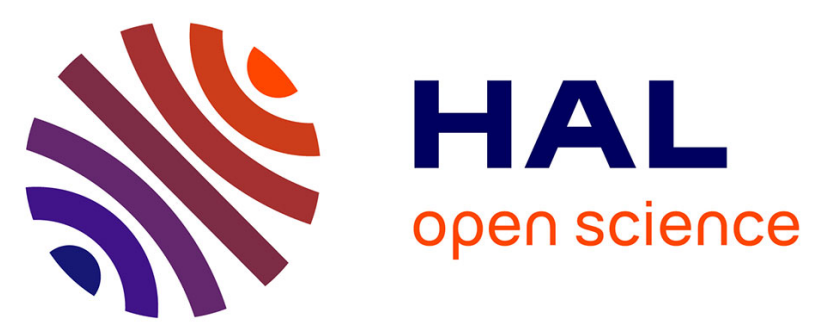

\title{
First results from SAM-FP: Fabry-Perot observations with ground-layer adaptive optics - the structure and kinematics of the core of 30 Doradus
}

C. Mendes De Oliveira, P. Amram, Bruno C. Quint, S. Torres-flores, R. Barba, D. Andrade

\section{To cite this version:}

C. Mendes De Oliveira, P. Amram, Bruno C. Quint, S. Torres-flores, R. Barba, et al.. First results from SAM-FP: Fabry-Perot observations with ground-layer adaptive optics - the structure and kinematics of the core of 30 Doradus. Monthly Notices of the Royal Astronomical Society, 2017, 469 (3), pp.34243443. 10.1093/mnras/stx976 . hal-01678526

\section{HAL Id: hal-01678526 https://hal.science/hal-01678526}

Submitted on 15 May 2018

HAL is a multi-disciplinary open access archive for the deposit and dissemination of scientific research documents, whether they are published or not. The documents may come from teaching and research institutions in France or abroad, or from public or private research centers.
L'archive ouverte pluridisciplinaire HAL, est destinée au dépôt et à la diffusion de documents scientifiques de niveau recherche, publiés ou non, émanant des établissements d'enseignement et de recherche français ou étrangers, des laboratoires publics ou privés. 


\title{
First results from SAM-FP: Fabry-Perot observations with ground layer adaptive optics - The structure and kinematics of the core of 30 Doradus
}

\author{
C. Mendes de Oliveira, ${ }^{1 \star}$ P. Amram,${ }^{2}$ Bruno C. Quint,${ }^{3}$ S. Torres-Flores, ${ }^{4}$ \\ R. Barbá ${ }^{4}$ and D. Andrade ${ }^{1}$ \\ ${ }^{1}$ Instituto de Astronomia, Geofísica e Ciências Atmosféricas da U. de São Paulo, Cidade Universitária, 05508-900, São Paulo, SP, Brazil \\ ${ }^{2}$ Aix Marseille Université, CNRS, LAM (Laboratoire d'Astrophysique de Marseille), Marseille, France \\ ${ }^{3}$ SOAR Telescope, AURA-O, Colina El Pino S/N, Casila 603, La Serena, Chile \\ ${ }^{4}$ Departamento de Física y Astronomía, Universidad de La Serena, Av. Cisternas 1200, La Serena, Chile
}

Accepted XXX. Received YYY; in original form ZZZ

\begin{abstract}
The aim of this paper is to present the first data set obtained with SAM-FP, a Fabry-Perot instrument mounted inside the SOAR telescope Adaptive-Optics Module. This is the only existing imaging Fabry-Perot interferometer using laser-assisted ground layer adaptive optics. SAM-FP was used to observe the ionised gas, traced by $\mathrm{H} \alpha$, in the centre of the 30 Doradus starburst (the Tarantula Nebula) in the LMC, with high spatial $(\sim 0.6$ arcsec, or $0.15 \mathrm{pc})$ and spectral $(R \simeq 11200)$ resolution. Radial velocity, velocity dispersion and monochromatic maps were derived. The region displays a mix of narrow, $\sigma \sim 20 \mathrm{~km} \mathrm{~s}^{-1}$ profiles and multiple broader profiles with $\sigma \sim$ $70-80 \mathrm{~km} \mathrm{~s}^{-1}$, indicating the complex nature of the nebula kinematics. A comparison with previously obtained VLT/FLAMES spectroscopy demonstrates that the data agree well in the regions of overlap but the Fabry-Perot data are superior in spatial coverage. A preliminary analysis of the observations finds a new expanding bubble South of R136, with a projected radius of $r=5.6 \mathrm{pc}$ and an expansion velocity of $29 \pm$ $4 \mathrm{~km} \mathrm{~s}^{-1}$. In addition, the first-time detailed kinematic maps derived here for several complexes and filaments of 30 Doradus allow identification of kinematically independent structures. These data exemplify the power of the combination of a high order Fabry-Perot with a wide-field imager $\left(3 \times 3 \operatorname{arcmin}^{2}\right.$ GLAO-corrected field of view) for high-resolution spatial and spectral studies. In particular, SAM-FP data cubes are highly advantageous over multi-fiber or long-slit data sets for nebula structure studies and to search for small-scale bubbles, given their greatly improved spatial coverage. For reference, this paper also presents two appendices with detailed descriptions of the usage of Fabry-Perot devices, including formulas and explanations for understanding Fabry-Perot observations.
\end{abstract}

Key words: Instrumentation: interferometers, spectrographs - ISM: bubbles, kinematics and dynamics, Hiı regions - (galaxies:) Magellanic Clouds

\section{INTRODUCTION}

This paper introduces a new restricted-use instrument ${ }^{1}$ for the SOAR telescope, or more precisely, it describes a new

\footnotetext{
^ E-mail: claudia.oliveira@iag.usp.br
} 
mode inside an existing facility instrument. It consists of a Fabry-Perot device mounted inside the SOAR Adaptive Module (SAM) with the SAM imager (SAMI, the existing imager, regularly used with SAM), to deliver high spatial resolution data cubes, in the optical, with a field of view of 3 $\mathrm{x} 3 \operatorname{arcmin}^{2}$. Presently, there is one Fabry-Perot instrument available for regular use with SAM-FP, which yields $R \simeq$ 11200 at $\mathrm{H} \alpha$. This new mode is hereafter referred to as SAMFP.

In Mendes de Oliveira et al. (2013) a number of recent Fabry-Perot spectrometers available for astronomy were described, but all of them were seeing-limited with a spatial sampling that depended on the detector pixel scale. FabryPerot instruments have been used for a range of sciences but the study of the internal kinematics of emission-line galaxies has been a major field of interest (e.g. Torres-Flores et al. 2013b, 2014; Alfaro-Cuello et al. 2015). Here, we highlight the potential for various studies, of the SAM-FP instrument, with a pixel size of $0.045 \mathrm{arcsec} / \mathrm{pixel}$ and ground layer adaptive optics (GLAO) correction down to 0.3 arcsec. A high-order, high-resolution Fabry-Perot with GLAO can be a unique tool in the study of kinematics of emission-line objects such as star forming galaxies, gas flows, planetary nebulae, HH objects, giant HII regions, among others. A tunable filter with GLAO, on the other hand, can be used for studying active galactic nuclei and quasars, outflows, massive young stars, mass loss processes and Ly $\alpha$ detection studies, to give a few examples. In this paper we focus on data taken with a high-order, high-resolution Fabry-Perot device. In a future paper, data taken with SAM-FP and a low-order tunable filter will be described.

We present Fabry-Perot data cubes for the largediameter expanding structures around a giant HiI region, the centre of 30 Doradus starburst (or 30 Doradus giant star forming region) also called the Tarantula Nebula, in the Large Magellanic Cloud. The study of shells and bubbles, due to stellar winds and supernova explosions, within giant HiI regions is crucial for understanding the processes of mass and energy transfer from the stellar component to the interstellar medium and the processes of triggering of star formation. Although kinematic studies of super-giant shells and super-bubbles in giant HiI regions are common in the literature, carried out both with long slit and more recently with 3D spectroscopy, they have not had the necessary coverage and spatial resolution to answer a few key questions. Even the origin of the supersonic motions present in these regions is still a matter of debate (Melnick et al. 1999). Moreover, it is still not understood if the kinematics of giant His regions are due to an underlying diffuse component or to superposed multiple kinematic components. Having high spatial and spectral resolution velocity maps, as those delivered by SAM-FP, is crucial to tackle these problems.

A few authors have studied the kinematics of the ionized gas in the 30 Doradus nebula, e.g. Chu \& Kennicutt (1994), Melnick et al. (1999), Redman et al. (2003), Torres-Flores et al. (2013) and references therein. All of these studies reported broad and possibly multiple components widespread in the nebula, which indicate a complex structure. These

search groups. Scientists outside the team are welcome to use this mode and, if interested, should contact the first author. studies were either performed (i) with long-slit echelle spectroscopy (e.g. Chu and Kennicutt 1994; Melnick et al. 1999), probing motions and physical conditions in small (pc) and larger (tens of pcs) scales but covering only a limited number of lines of sight or (ii) with fibers, as the work of TorresFlores et al. (2013), with a somewhat larger field of view, but probing the nebula in a sparse manner (every $5 \times 5 \mathrm{pc}^{2}$, with a filling factor of 0.0028). The advantage of the present study is that it combines 3D spectroscopy information with continuous coverage of the field over a fairly large field of view, with a pixel size (binned $4 \times 4$ ) of $0.18 \times 0.18 \operatorname{arcsec}^{2}$ (or $0.045 \times 0.045 \mathrm{pc}^{2}$ ). These data show that the kinematic profiles change on scales of a tenth of a parsec over the whole field. The wealth of details seen in Hubble Space Telescope (HST) images of nearby giant HII regions can now be investigated using similar quality kinematic data taken with SAM-FP with a laser guide star.

The main purpose of this paper is to highlight the potential of Fabry-Perot maps obtained with adaptive optics in the study of 30 Doradus and similar giant HiI regions. A high-order Fabry-Perot was used to observe the ionized gas within 30 Doradus, in its strongest optical line: $\mathrm{H} \alpha$. In section 2, we first describe the new mode of SAM with a Fabry Perot, SAM-FP. We also include in this section a general description of the data reduction. The observations are presented in section 3. Section 4 compares the present observations with earlier ones taken with a different instrument. We describe in section 5 our results which include monochromatic, radial velocity, velocity dispersion and channel maps of the region. We identify a new expanding bubble in the centre of 30 Doradus and we describe the overall kinematic features observed, thanks to the high spatial resolution and continuous spatial coverage of the data. Section 6 summarises our main results. Appendix A details all steps necessary for taking Fabry-Perot observations. Appendix B provides further information on section 2.2 and Appendix A. The Fabry-Perot guide for observers given in the appendices is essential because s uch information cannot be found in one place anywhere else, and this often forms a barrier to using Fabry-Perot instruments. The main aims of this paper are, therefore, to describe the SAM-FP instrument, to present example observations taken with this instrument and finally to provide recommendations on how to best perform Fabry-Perot observations, with any generic FP instrument.

At the adopted distance of $50 \mathrm{kpc}$ to 30 Doradus, 4 arcsecs corresponds to $1 \mathrm{pc}$.

\section{DESCRIPTION OF THE INSTRUMENT}

\subsection{SAM-FP: a new mode for SAM}

The SAM instrument (Tokovinin et al. 2008) is a unique facility instrument mounted on the $4.1 \mathrm{~m}$ SOAR telescope at Cerro Pachón. SAM improves image quality by partial correction of turbulence near the ground using ground-layer adaptive optics (Tokovinin et al. 2010, Tokovinin et al. 2012). This instrument can feed corrected images either to a visitor instrument or to the internal wide-field optical imager, SAMI (Fraga et al. 2013). SAMI works with a single $\mathrm{e} 2 \mathrm{v}$ CCD with $4096 \times 4112$ pixel $^{2}$. Each pixel is $15 \times 15 \mu \mathrm{m}^{2}$ 
square, the pixel scale is 45.4 mas and the total field of view is $3 \times 3 \operatorname{arcmin}^{2}$. The CCD is operated with the SDSU-III controller, which reads the full unbinned chip in 10 seconds with a noise of 3.8 electrons (without patterns) and a gain of 2.1 electrons/ADU. SAM can provide very sharp images with image quality as good as $\sim 0.3$ arcsec in the r-band, in favourable conditions, after laser corrections. SAMI is regularly mounted on SAM for use of the SOAR community and has been used with success (e.g. Fraga et al. (2013)).

SAM was built with space in the collimated beam for the installation of an etalon, to enable future Fabry-Perot observations, which had not been implemented until now. Presently, there is one Fabry-Perot instrument fully tested for use in SAM, with a pupil size of $65 \mathrm{~mm}$ (ICOS ET$\left.65^{2}\right)$. It has a mean gap of $200 \mu m$ and, at $\mathrm{H} \alpha 6562.78 \AA$, an interference order $p \simeq 609$. For the observational run described in this paper, an effective finesse $F \simeq 18.5$ and a resolution $R \simeq 11200$ (at $\mathrm{H} \alpha$ ) were measured; however these values may change slightly depending on the accuracy of the Fabry Perot plates' parallelism. The Fabry-Perot plates have a broadband coating covering the range $400-700 \mathrm{~nm}$ with a reflectivity of $90 \%$. This coating was inspected in 2015 by ICOS and was found to be in good condition.

The etalon is controlled by a CS100 controller (Serial Number 8030), borrowed from the Anglo Australian Observatory. The controller is attached to the SOAR's Nasmith cage by a mechanical adaptor specifically built for it, placed in such a way that one can access and adjust it while simultaneously inspecting the etalon. The computer that runs the CS100 is located on one side of the Nasmith platform where SAM is permanently mounted. The CS100 contains two channels, $\mathrm{X}$ and $\mathrm{Y}$, to control the parallelism between the Fabry-Perot plates, and a $\mathrm{Z}$ channel to control the spacing. A similar CS100 to the one used here is described in detail by Veilleux et al. (2010).

SAM's original concept already included a mechanism to put the Fabry-Perot in and out of the beam, which can now be used when doing Fabry-Perot observations. It was necessary to design and fabricate a mechanical plate adaptor in order to mount the Fabry-Perot device inside SAM. This adaptor can be removed at any time but it is now regularly placed into SAM. It was devised in a way to allow the Fabry-Perot to be easily placed in and out of the instrument, facilitating the task of checking the parallelism while tuning the CS100, which is located immediately beside it.

\subsection{Data Acquisition}

The existing control software of SAMI was modified (from a module previously written for the Brazilian Tunable Filter Imager, Mendes de Oliveira et al. 2013) in order to create a script for taking a scanning sequence. The script was used during the setup of the Fabry-Perot, for on-sky observations, as well as for acquisition of spectral calibrations. The reasons for each command and descriptions of how the Fabry-Perot

2 This was fabricated in 1988 by Queensgate, nowadays called IC optical Systems (ICOS), for the Anglo Australian Observatory and it is, presently, in an extended loan to the University of São Paulo, kindly made possible by the present director Dr. Warrick Couch and the previous director Dr. Matthew Colless. parameters are actually determined are described in the appendices. In summary, the script allows computing the exact value for the free spectral range of the Fabry-Perot device, finds the ideal number of steps needed to scan the free spectral range and defines a scanning sequence. As for a grism, the wavelength range and the spectral resolution are the important parameters that define a Fabry-Perot device. However, for a Fabry-Perot, the effective spectral range is in fact the free spectral range (FSR), see equation (2) below, and the spectral resolution depends on the interference order $\mathrm{p}$ and the effective finesse F, which follows relation (4) below.

\subsubsection{Determining the free spectral range, the effective finesse and spectral resolution}

In order to compute the FSR at a given wavelength $\lambda$, the interference order $\mathrm{p}$ should be known and it is linked to the other quantities by the basic Fabry-Perot equation:

$\mathrm{p}=\frac{2 \mathrm{ne} \cos \theta}{\lambda}$

where $\mathrm{n}\left(\mathrm{T}=0^{\circ} \mathrm{C}, \mathrm{P}=1 \mathrm{~atm}\right) \simeq 1.0003$ is the index of the air layer between the plates of the Fabry-Perot device, e the inner separation between the coated plates, $\theta$ the incidence angle and $\lambda$ the wavelength. This is valid in the case where the so called additional phase-lag $\psi(\lambda)$, defined in the appendix, can be neglected; see section B1 and equation B1 for the general case. From equation (1), it follows that for a given $\mathrm{n}$, e and $\theta$, the FSR at the interference order $\mathrm{p}$ and at the wavelength $\lambda$ can be computed by:

$\Delta \lambda_{\mathrm{FSR}}=\frac{\lambda}{\mathrm{p}}\left(\frac{1}{1-\left(1 / \mathrm{p}^{2}\right)}\right)$

Conversely, for a given $\mathrm{n}, \lambda$ and $\theta$, again from equation (1), the FSR at the interference order $\mathrm{p}$ and at the inner separation of the plates e can be computed by:

$\Delta \mathrm{e}_{\mathrm{FSR}}=\frac{\mathrm{e}}{\mathrm{p}}=\frac{\lambda}{2 \mathrm{n} \cos \theta}$

$\Delta \lambda_{\text {FSR }}$ is then the FSR in $\AA$ (if $\lambda$ is given in $\AA$ ), which can be easily related to the FSR in velocity units (see numerical values below), while $\Delta \mathrm{e}_{\mathrm{FSR}}$ is the increment of plate separation necessary to scan the FSR.

As described in the previous section, one Fabry-Perot device is presently available, ICOS ET-65, with a high interference order $\mathrm{p} \simeq 609$, at $\mathrm{H} \alpha$, allowing coverage over a FSR $\simeq 10.8 \AA$ (following equation 2 ), which corresponds to $\simeq 492$ $\mathrm{km} \mathrm{s}^{-1}$.

In order to select only one or a few interference orders, an interference filter selecting a passband broader than the FSR has to be placed in the optical path, generally at (or close to) the focal plane to avoid wavelength dependence. Ideally the central wavelength of the interference filter should correspond to the mean velocity of the source. For instance, for the observations of 30 Dor with a velocity restframe of $V_{s}=267 \mathrm{~km} \mathrm{~s}^{-1}$ (Torres-Flores et al. 2013, placing $\mathrm{H} \alpha$ at $\lambda=6568.65 \AA$ ), we selected an interference filter centred at $\lambda_{c}=6568 \AA$ having a passband of $19 \AA$ (which is, in the case of the Fabry Perot used here, almost twice the FSR).

The resolving power, also called spectral resolution $\mathrm{R}_{\lambda}$ 
(or simply R) of the Fabry-Perot is provided by the following equation:

$\mathrm{R}_{\lambda}=\mathrm{pF}$

We thus need to know the interference order $\mathrm{p}$ and the effective finesse F. In practice, both parameters are roughly known and they are given by the manufacturer. The effective finesse needs nevertheless to be measured with accuracy. As explained in the end of Section B3.3, the effective finesse can be simply obtained dividing the FSR by the width of an arc line (e.g. the Ne I emission line $6598.95 \AA$ ) in a spectrum extracted from the calibration cube. The spectral resolution can be inferred from the width of a calibration arc line. The high order Fabry-Perot for use with SAM-FP has $\mathrm{p} \simeq 609$, at $\mathrm{H} \alpha 6562.78 \AA$. During the observational run when data for this paper was obtained, the effective finesse of the FP was measured to be $\mathrm{F} \simeq 18.5$, providing a spectral resolution of $\mathrm{R} \simeq 11200$. Further information on the nature and on the determination of the effective finesse is provided in section B3.3.

\subsubsection{Taking calibrations and on-sky data}

Once we know the FSR and effective finesse, then the optimal number of steps to scan a FSR has to be computed and the scanning sequence has to be defined. For the observations of 30 Doradus described in this paper, we decided to slightly oversample by $5-10 \%$, choosing a scanning sequence of 40 steps with a scanning step of $\sim 12.8 \mathrm{~km} \mathrm{~s}^{-1}$. The same number of channels must be used for on-sky and calibration scanning sequences, except for the one calibration cube that is obtained for determination of the FSR and effective finesse, which should be highly oversampled and should cover more than one FSR (see Appendix B).

Spectra of calibration arc lamps (those from the SOAR calibration unit) need to be taken in day time, ahead of the first observing night, for wavelength calibration and for computation of the several Fabry-Perot parameters that will define the observation strategy. Suitable interference filters should be illuminated by the calibration lamps, so that the final spectra will contain at least one arc line with similar wavelength to that of the observed emission-line ( $\mathrm{H} \alpha$ in our case, redshifted due to the velocity of the target). In our case, we chose to use the Ne I $6598.95 \AA$ emission line for calibration and the best option was then to use the interference filter BTFI 6600.5/19.3, available from the BTFI filter set.

A reference wavelength calibration has to be repeated at the position of the object on the sky ideally at the start and at the end of each observation to account for possible mechanical flexures of the instrument as well as changes in the air index inside the FP plates due to temperature, pressure and humidity changes along the night. In section 2.3 details are given about how to perform wavelength calibration of the data.

We described above and in the Appendices how to compute the parameters necessary for setting up the Fabry-Perot and taking data. In practice, a Python program was written in order to define the acquisition sequence for a Fabry Perot data cube. It produces a shell script that has to be run each time the observer wants to take a on-sky observation or a calibration. The Python program is available in the
SAMI machine and upon request. It is self explanatory and easy to use. The input parameters to the script are given in Appendix A.

We should note that a feature was included in the control software of SAMI in order to allow pausing/stopping while an exposure is being taken. This was necessary, given that there are laser interruptions that are mandatory during a given night. In addition, the observation can be paused/stopped due to passing clouds. When the stop or pause button is pressed, the ongoing exposure is then finished and read out before the action (pause or stop) takes place.

A mode allowing $8 \times 8$ binning was needed for testing, at the commissioning, and this was then included in the software. A $4 \times 4$ binning mode (an option that already existed previously in SAMI but had not been implemented) was included in the new control software and has been used for the Fabry-Perot observations presented in this paper. Indeed, a binned pixel of size 0.18 arcsec still allows a good sampling of the best seeing and considerably increases the signal-to- noise ratio (SNR) per unit surface.

\subsection{Data Reduction}

The raw images obtained with SAM-FP are transformed into scientifically useful data cubes after following several procedures described below. Each FITS file obtained with SAMI has four extensions (the CCD controller has four amplifiers). Before the combination of the four fits extensions into one fits image, we estimate the background using the overscan region by fitting a 3rd degree polynomial along the columns' direction and subtracting it from each column. This procedure is followed for all data including bias, dark, flat, calibration and science files. After combining the extensions we then subtract the bias from the science frames and correct them by the normalised flats. It is important to note that SAMI's CCD has a strong instrumental feature that is not always present but can greatly affect the images. These are described as "arcs" in internal SAMI documents. They can be removed from the science images by subtracting suitably scaled dark images from the science frames, before flat fielding. It is, therefore, highly recommended that at least 10-20 darks are taken per night, with exposure times of 3-10 minutes, for this purpose. This feature was not seen in the data presented here, so no correction was needed. For science images with exposure times longer than several seconds, it is important to do cosmic ray cleaning. In our case, all procedures described above were performed using $\operatorname{IRAF}^{3}$, except for the procedure of combination of the fits extensions, performed by a program kindly provided by Andrei Tokovinin.

The final calibration and science images were then converted into data cubes. Having the calibration cube (in our case the Ne I data cube) in hand, the phase correction was performed with the main goal of re-arranging the spectral information in the science data cube. As it is well known, the spectral information in each pixel, for Fabry-Perot data,

3 IRAF is distributed by the National Optical Astronomy Observatories, which are operated by the Association of Universities for Research in Astronomy, Inc., under cooperative agreement with the National Science Foundation. 
Table 1. Journal of Perot-Fabry observations

\begin{tabular}{|c|c|c|}
\hline \multirow[t]{6}{*}{ Observations } & Telescope & SOAR \\
\hline & Instrument & Fabry-Perot inside SAM \\
\hline & UT Date & March $18^{\text {th }} 2015$ \\
\hline & FWHM of stars (laser corrected) & $\sim 0.5-0.7 "$ \\
\hline & Mean scanning lambda of 30 Doradus obs & $6568.78 \AA$ \\
\hline & 30 Doradus systemic velocity & $267 \mathrm{~km} \mathrm{~s}^{-1}$ \\
\hline \multirow[t]{3}{*}{ Interference Filter } & Central Wavelength & $6568 \AA$ \\
\hline & FWHM & $19 \AA$ \\
\hline & Transmission & 0.70 \\
\hline \multirow[t]{3}{*}{ Calibration } & Ne I reference line & $6598.95 \AA$ \\
\hline & Central lambda of filter used to select Ne line & $6600.5 \AA$ \\
\hline & Width of filter used to select Ne line & $19.3 \AA$ \\
\hline \multirow[t]{5}{*}{ Fabry-Perot } & Company and ID & ICOS ET-65 \\
\hline & Interference Order & $609 @ 6562.78$ \\
\hline & Free spectral range at $\mathrm{H} \alpha$ & $10.8 \AA\left(492 \mathrm{~km} \mathrm{~s}^{-1}\right)$ \\
\hline & Effective finesse & 18.5 at $6598.95 \AA^{c}$ \\
\hline & Spectral resolution at $\mathrm{H} \alpha$ & $11200^{c}$ \\
\hline \multirow[t]{2}{*}{ Sampling } & Number of Scanning Steps & $40^{c}$ \\
\hline & Sampling Step & $0.28 \AA\left(12.8 \mathrm{~km} \mathrm{~s}^{-1}\right)^{c}$ \\
\hline \multirow[t]{5}{*}{ Detector } & $\mathrm{e} 2 \mathrm{v}$ & CCD $4096 \times 4112$ \\
\hline & Total Field of View & $120 " \times 120 " a$ \\
\hline & Pixel size & $0.18 " b$ \\
\hline & Readout noise (unbinned) & 3.8 electrons \\
\hline & Gain & 2.1 electrons/ADU \\
\hline \multirow[t]{2}{*}{ Exposures times } & Total on-target exposure & 80 minutes \\
\hline & Total exposure time per channel & $120 \mathrm{~s}$ \\
\hline
\end{tabular}

$a$ The field of view is limited to $2 \times 2 \operatorname{arcmin}^{2}$ by the interference filter used.

$b$ The pixel size of SAMI is 0.045 arcsec. Pixels were binned $4 \times 4$.

$c$ These values may change slightly from one observational run to the next, given that they depend on the accuracy of the FP plates' parallelism (see appendix) and this has to be checked every time the instrument is used.

is radially shifted with respect to the next, as one moves in the cube, along the spatial direction. This shift follows a parabolic surface centred on the Fabry-Perot optical center. In addition, because Fabry-Perot data are periodic, the spectrum repeats itself. Three steps are required for the phase correction: phase-map extraction, fitting and application. These steps have already been described previously in the literature, e.g. Atherton et al. (1982), Bland et al. (1987), Amram et al. (1989). After phase correction we then have a data cube where each frame represents one wavelength (modulo the FSR, given the cyclic nature of the Fabry-Perot device).

For wavelength calibration we use two calibration cubes taken at different wavelengths. In fact, only one cube would be necessary for calibration of data taken with a high-order Fabry Perot but a second one can be useful for interference order checking. In our case we use a Ne Lamp with two filters that contained the following lines respectively, $6598.95 \AA$ (BTFI filter 6600.5/19.3) and 6717.04 $\AA$ (BTFI filter $6745.5 / 38.6$ ), but the latter is only for order checking. For that, the calibration cube (in our case the $6598.95 \AA$ data cube) has to cover more than one free spectral range so that the line will appear in two different orders and the second cube (in our case the $6717.04 \AA$ data cube) can cover one free spectral range only. We also need to extract a 1D spectrum from these cubes at the centre of the rings. Section 5.9 of Atherton et al. (1982) describes in detail the procedure for wavelength calibration of tunable filter images that has been adapted for our use and built into a script. After wavelength calibration the cube is then ready for night-sky line subtraction and derivation of the monochromatic, velocity and dispersion maps.

All command line scripts needed to perform the tasks described above are available at the SOAR observatory, with supporting documentation.

\section{OBSERVATIONS OF 30 DORADUS}

The observations of 30 Doradus, centered at RA 053847.4 and Declination -6905 43, were taken on UT date March 18th, 2015, with the high-resolution scanning Fabry-Perot interferometer described above, placed inside SAM, fed to SAMI. Although the total field of view of the instrument is $3 \times 3 \operatorname{arcmin}^{2}$, due to the smaller size of the $\mathrm{H} \alpha$ interference filter, the useful field of view was $2 \times 2 \operatorname{arcmin}^{2}$. We used a $2 \times 2$ inch $^{2}$ interference filter from the Cerro Tololo list, centred at $6568 \AA$ (filter 6568-20), with a FWHM of $19 \AA$ and with transmission of $\sim 70 \%$ (according to the list of CTIO filters). The free spectral range of the interferometer (492 $\mathrm{km} \mathrm{s}^{-1}$ ) was covered in 40 scanning steps. The setup and observing details are summarized in Table 1.

The observations were taken under photometric conditions. The seeing during the observations varied between 0.7 and 0.8 arcsec. GLAO correction lead to an improvement of spatial resolution and flux of up to $\simeq 2$, yielding a FWHM of $0.5-0.7$ arcsec. The spatial resolution of the cubes was then determined by the laser corrected FWHM with a mean value of 0.6 arcsec or 0.15 pc. The instrumental profile, as 


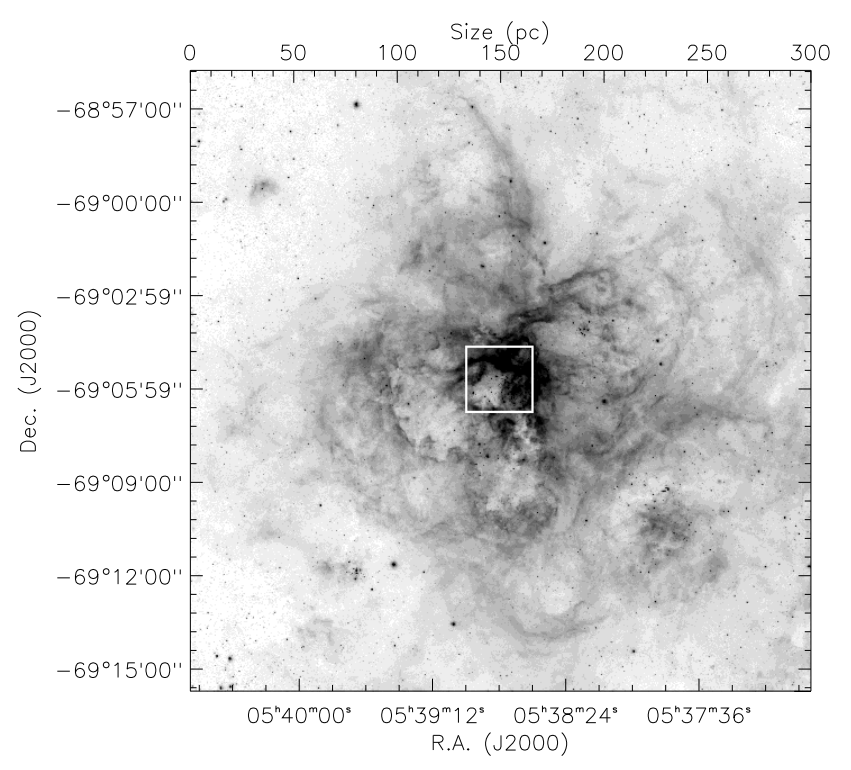

Figure 1. H $\alpha$ image taken at the T80-South telescope during comissioning of its wide-field camera, with images with a field of view of $1.4 \times 1.4$ degree $^{2}$. The large image above is a subset of it, covering a $\sim 20 \times 20$ arcmin $^{2}$ field centred on R136 and the white box indicates the central $2 \times 2 \operatorname{arcmin}^{2}$ of the image observed with SAM-FP.

measured from Ne calibration lamp lines was $0.586 \AA$ or 26.8 $\mathrm{km} \mathrm{s}^{-1}$, which corresponds to a spectral resolution of about 11200 at $\mathrm{H} \alpha$. The last four channels were taken after a laser interruption of a few minutes and after some technical problems which took about 45 minutes to be fixed. The data for the last channels were taken under higher airmass $(>1.5)$ and slightly worse seeing ( 0.7 arcsec after correction). This, however, represented only $10 \%$ of the data and it did not degrade significantly the quality of the cube as a whole.

Fabry-Perot observations are usually taken with short exposure times and in a number of sweeps, to account for transparency and seeing variations. However, given that we are observing with a classical CCD (not an EMCCD nor a photon-counting camera), we chose to make just one sweep of 40 channels and with fairly long exposure times of 120 seconds each, to minimize the readout noise (see also section A3.4). The night sky lines have been identified by plotting a histogram of wavelengths and picking the most frequent values (given that the sky lines are present in every pixel). Knowing their wavelengths and intensities, night sky lines were then subtracted. Alternatively, a nebulae-free data cube beside the object (new pointing) could have been observed to directly subtract the night sky lines (see section A4.2) but this was not done in the case of these 30 Doradus observations.

Figure 1 shows the region observed in the present study. The background image is an $\mathrm{H} \alpha$ observation taken during commissioning of the robotic telescope T80-South ${ }^{4}$. A cut

4 T80-South is an 80-cm telescope mounted at Cerro Tololo, which has a CCD camera with a $9.2 \mathrm{k} \times 9.2 \mathrm{k}$ pixel $^{2} \mathrm{CCD}$ yielding a $1.4 \times 1.4$ degree $^{2}$ field of view (with 0.55 -arcsec pixels). The telescope was built by the companies AMOS and ASTELCO and the CCD camera by Spectral Instruments. of $20 \times 20 \operatorname{arcmin}^{2}$ centred on 30 Doradus was extracted from the T80-South image and it is shown in Figure 1. The area of $2 \times 2 \operatorname{arcmin}^{2}$, observed with the Fabry-Perot, is indicated with a white box. The bright star cluster R136 is included in the area observed.

\section{DATA ANALYSIS}

\subsection{Comparing Fabry-Perot data with previously published $\mathrm{H} \alpha$ kinematic data of 30 Doradus}

The Fabry-Perot $\mathrm{H} \alpha$ data cube of 30 Doradus was compared with a data cube obtained with the FLAMES instrument at VLT and the Giraffe spectrograph in MEDUSA mode, published by Torres-Flores et al. (2013). About 900 fibers were used to obtain kinematic information over a field of view of $10^{\prime} \times 10^{\prime}$, centred on R136, with a spectral resolution of $R \sim 17000$ (compared to $R \simeq 11200$ for the Fabry-Perot data). The distance between each fiber for the FLAMES/VLT setup was 20 arcsec. In the case of the FabryPerot data, we observed a field of view of 2 ' $\times 2$ ', with a pixel scale of 0.18 arcsec and with a median FWHM of 0.6 arcsec. Therefore, the spectral resolution of the Fabry-Perot data is about $30 \%$ poorer but the spatial coverage or spatial resolution (or more precisely, the number of independent measurements) is more than $30^{2}$ times higher than that for the VLT data (which has a measurement taken with a MEDUSA fiber of $1.2 \operatorname{arcsec}$ diameter at every 20 arcsec). For this reason, and in order to do a fair comparison, we have extracted from the Fabry-Perot data, squared regions of 1.1 " $\times 1.1$ " that are placed at the same positions as the MEDUSA fibers were. For each region we have co-added the profiles, to get a mean $\mathrm{H} \alpha$ profile of the region, which can then be compared with the MEDUSA fiber data. The red profiles in Figure 2 show the FLAMES/VLT H $\alpha$ data taken from Torres-Flores et al. (2013) and the black profiles represent the Fabry-Perot data. All profiles have been normalised to the integrated flux of the line in each specific region or fiber. In both cases the background image corresponds to an $\mathrm{H} \alpha$ observation of 30 Doradus taken with the ESO NTT/EMMI instrument under program 70.C-0435(A).

Inspecting the profiles shown in Figure 2, narrow- and broad-emission-line profiles can be seen at different regions of the nebula. The narrow profiles are generally associated with $\mathrm{H} \alpha$ filaments. Broad profiles have been previously reported by Chu \& Kennicutt (1994) among other authors. The broadest profiles can be seen to the East of R136, and they may be associated with a cavity that has distinct identifications given by different authors, e.g. it is called region $H$ by Melnick et al. (1999) and Christmas Tree by De Marchi et al. (2014). It is a region with an additional extinction component as compared to the neighbouring regions and it resembles the silhouette of a Christmas tree.

Comparing the black and red lines in Figure 2 we are able to check if the Fabry-Perot observations reproduce well the VLT/FLAMES results. Despite having about $30 \%$ lower spectral resolution $(R=11200$ against $R=17000$, readily seen by the narrower widths of the VLT profiles), the Fabry-Perot $\mathrm{H} \alpha$ profiles clearly display quite similar shapes to the previously published data. For instance, from top left, boxes $(3,2),(3,3)$ and $(2,3)$ display the same blue component in 
both cases. Also, the shapes of the $\mathrm{H} \alpha$ profiles in boxes $(4,4)$, $(4,5)$ and $(5,4)$ are similar for both datasets. In addition, the general behaviour of the emission line profiles in the central region of the Christmas Tree is also consistent with results in the literature (e.g. Chu and Kennicutt 1994, Melnick et al. 1999).

There are, however, a few notable differences in the line profile shapes between the VLT and the Fabry Perot data cubes specially in the Southeastern region of the Christmas Tree, e.g. for box $(2,4)$. These differences are real, given that particularly in this region the profiles vary strongly in small scales. The great variation from one frame to the next in the region of the Christmas Tree, indicating very small scale fragmented velocity features, will be discussed in detail in Section 5.2 and in Figure 6.

In order to do a fair comparison between the SAM-FP and the VLT/FLAMES data cubes of 30 Doradus, we centred the integrated $\mathrm{H} \alpha$ emission line from the SAM-FP at the exact same radial velocity of the integrated $\mathrm{H} \alpha$ emission line profile of the VLT/FLAMES cube, over the region of overlap. The procedure undertaken to match the radial velocities of both data sets was the following. First, we derived a sub-cube of the VLT/FLAMES H $\alpha$ data cube, which covered the same $2 \times 2 \operatorname{arcmin}^{2}$ field of view of the SAM-FP observations (Figure 2, black lines). We derived the integrated $\mathrm{H} \alpha$ profile for this region, fitted a single Gaussian to it and obtained a central wavelength of $6568.72 \AA$, which corresponds to $270.7 \mathrm{~km} \mathrm{~s}^{-1}$. We note that this value is slightly higher than the radial velocity derived by Torres-Flores et al. (2013) of $267.4 \mathrm{~km} \mathrm{~s}^{-1}$, but that was for the whole VLT data cube $\left(10 \times 10 \operatorname{arcmin}^{2}\right)$. We then obtained the integrated $\mathrm{H} \alpha$ profile of the SAM-FP data cube, fitted a Gaussian to it and measured a central wavelength that we matched to $6568.72 \AA$. Therefore, the zero point of the radial velocity of our dataset is tied to that derived by Torres-Flores et al. (2013), for the same region.

Once we have compared both data sets and they show good general agreement, we proceed to analyse the FabryPerot data alone, for which we have continuous spectroscopic information with a scale of $0.18 \operatorname{arcsec}(0.045 \mathrm{pc})$.

\subsection{Fitting Gaussians to the $\mathrm{H} \alpha$ data cube}

We have fitted a single Gaussian to each observed $\mathrm{H} \alpha$ profile in 30 Doradus using the code FLUXER, an interactive routine in IDL. This uses the package MPFIT to fit a modelled Gaussian to the observations, which allows us to determine the center, width and flux of the Gaussian. In this case, the centre can give us information regarding the radial velocity of the gas and the width can be used to characterise the overall complexity of the $\mathrm{H} \alpha$ profiles, indicating where there may be a superposition of different kinematic structures or which regions may be affected by turbulence. In this manner we then computed the radial velocity and dispersion maps for the region.

In a few specific cases it was necessary to fit several Gaussians to the profiles, as it was the case, for example, when measuring the expansion velocity of a newly found bubble in the centre of 30 Doradus in section 5.3. For that, we used the code PAN (Dimeo 2005) to fit multiple (and also single) Gaussians to the observed profiles. This code, which runs in IDL, allows the user to define interactively
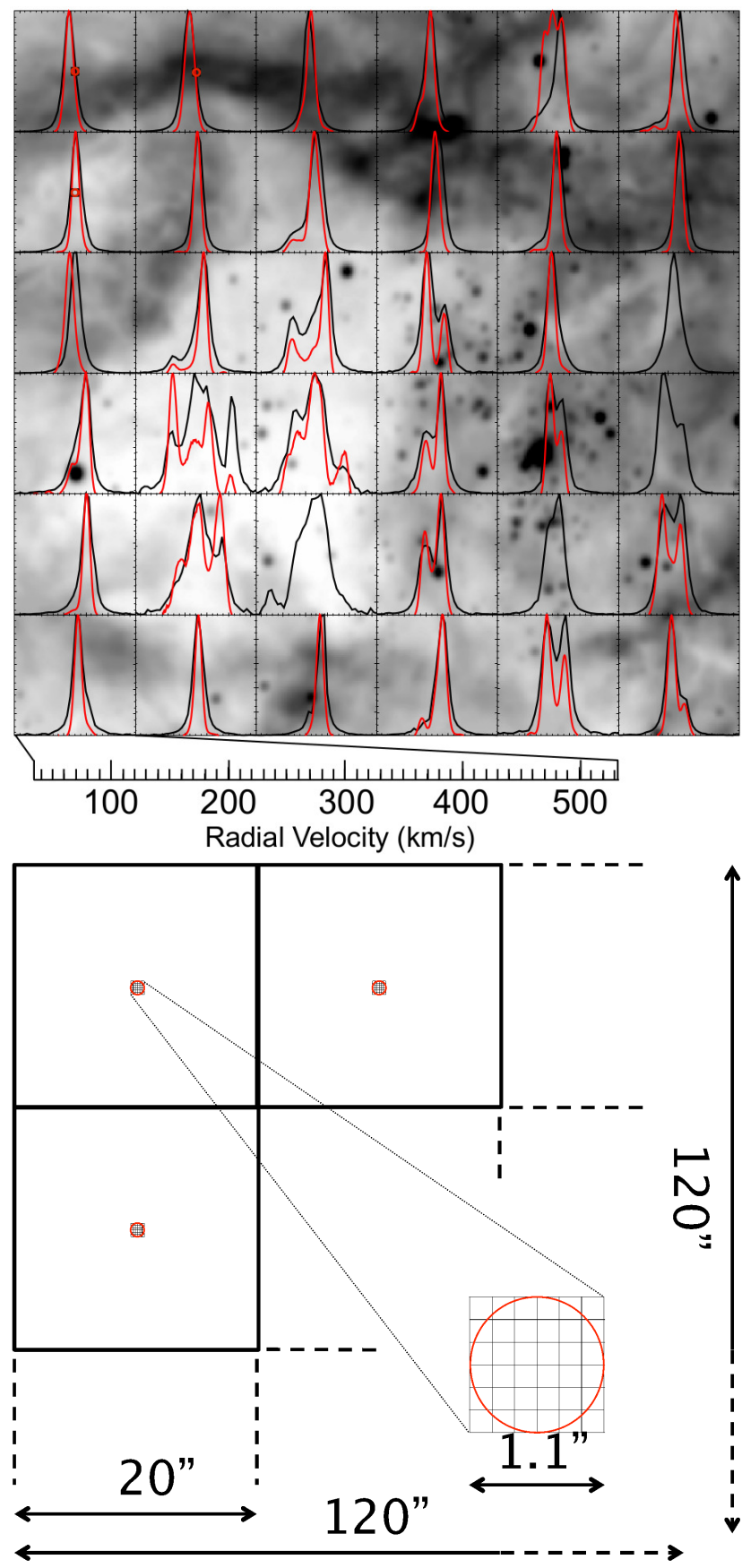

Figure 2. Upper panel: central region of 30 Doradus. The red profiles correspond to the $\mathrm{H} \alpha$ emission-line VLT data, extracted from fig. 3 of Torres-Flores et al. (2013). These correspond to the integrated spectrum within a fibre of 1.2 arcsec diameter, placed at the centre of each $20 \times 20 \operatorname{arcsec}^{2}$ box (the position of the fibre is depicted with a red circle in the three upper left boxes also see lower panel for the sketch representing the approximate fibre diameter). Boxes with no red profiles correspond to broken fibres. The black profiles correspond to the average profiles of the FP data extracted from a square aperture of $1.1 \times 1.1 \operatorname{arcsec}^{2}$ located at the centre of each box, in an attempt to mimic the VLT data (the $1.1 \times 1.1 \operatorname{arcsec}^{2}$ area within which the profiles were averaged is best seen in the sketch of the lower panel). The background image corresponds to an $\mathrm{H} \alpha$ image of 30 Doradus taken at ESO NTT (see text). The velocity scale is shown in one case, on the lower left, and it is the same for all profiles. Lower panel: sketch of the fields shown in the upper panel. 

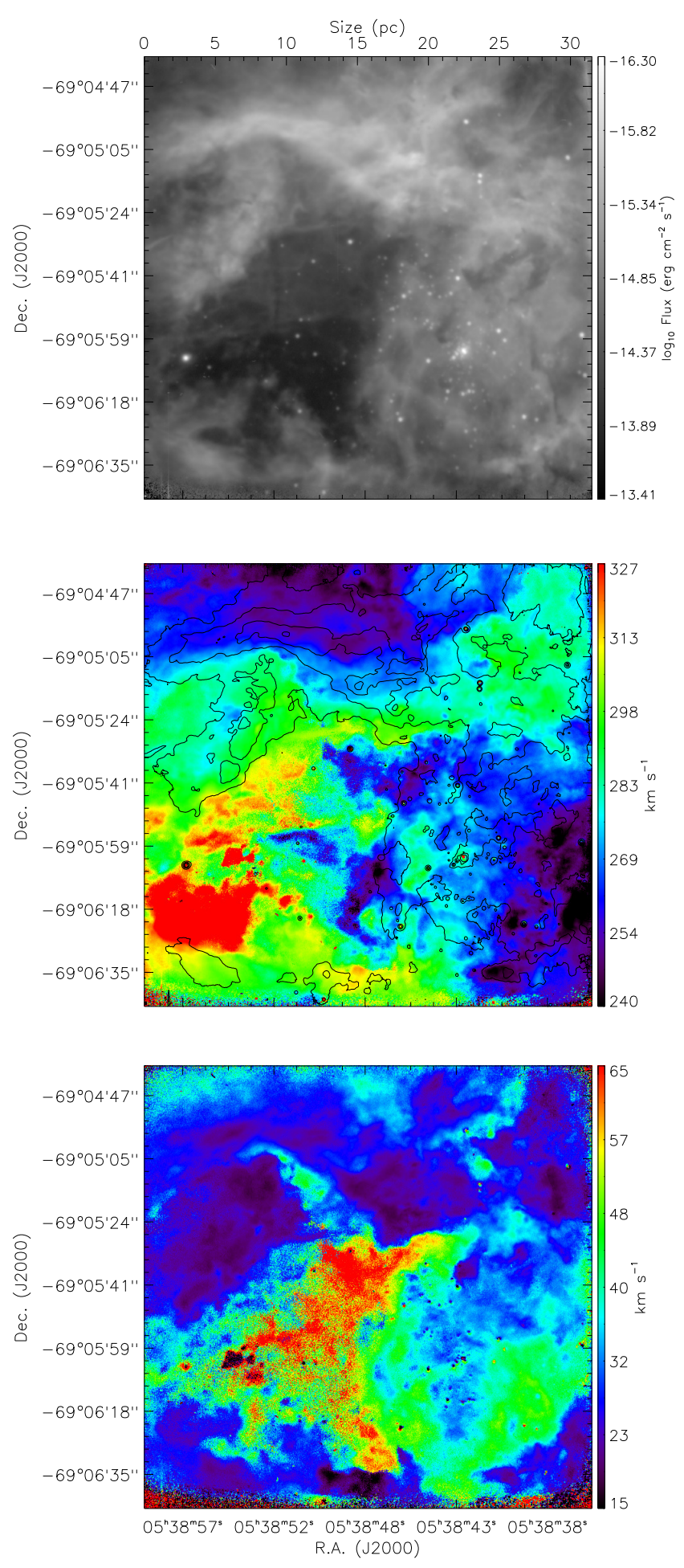

Figure 3. Monochromatic image, radial and dispersion velocity maps of 30 Doradus. Top: $\mathrm{H} \alpha$ monochromatic map derived from the Fabry-Perot data cube, as explained in the text. This map is flux calibrated (see Section 5.1). Middle panel: Velocity field for the same region as shown in the top panel. The scale is shown in $\mathrm{km} \mathrm{s}^{-1}$. The contours show the following flux levels (from the monochromatic map): $8 \times 10^{-16}, 2 \times 10^{-15}, 5 \times 10^{-15}, 8 \times 10^{-15}$ erg s $\mathrm{s}^{-1} \mathrm{~cm}^{-2}$. Bottom: Velocity dispersion map of the same region. The scale is shown in $\mathrm{km} \mathrm{s}^{-1}$. The map was corrected by instrumental and thermal widths.

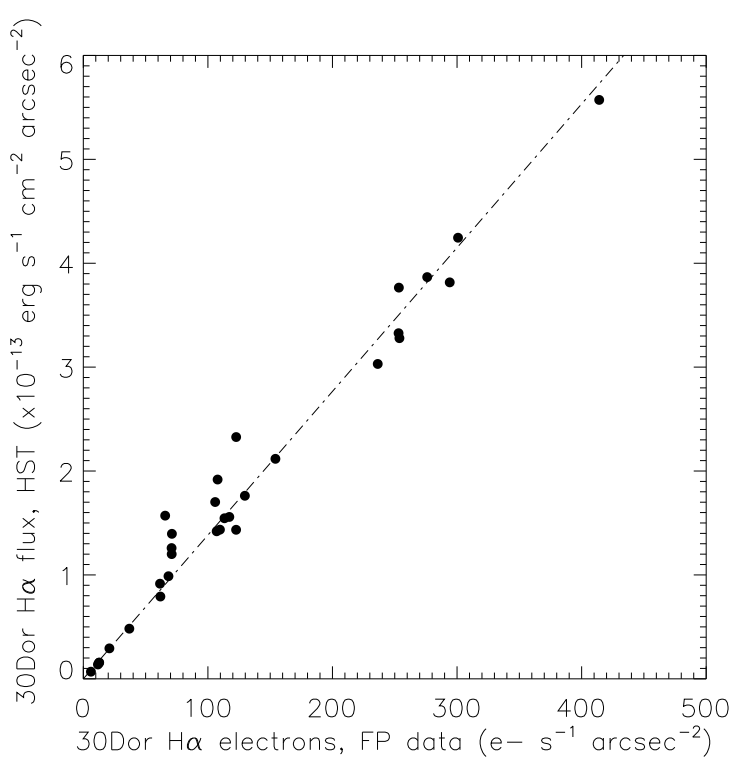

Figure 4. Flux calibration of the SAM-FP data of 30 Doradus using an archival HST image of the region. In the $\mathrm{x}$-axis instrumental fluxes of 31 regions located across the FP field, within an aperture of 3 arcsec in radius, are computed and plotted and in the $y$-axis the corresponding fluxes for the same regions obtained from the HST image are given.

multiple Gaussians, estimating the flux, width and centre of each component.

\section{RESULTS}

In this section we describe the main kinematic features found in the $\mathrm{H} \alpha$ Fabry-Perot data cube of 30 Doradus.

\subsection{H $\alpha$ map and flux calibration}

At first we attempted to create the monochromatic map of the 30 Doradus region by fitting a single Gaussian to each pixel. However, we concluded that the fit of single Gaussians to the often-encountered multiple $\mathrm{H} \alpha$ profiles brought no meaningful information to the monochromatic map, given the complex profiles of the nebula. The $2 \mathrm{D}$ collapsed data cube was then used as the $\mathrm{H} \alpha$ monochromatic map (shown in the top panel of Figure 3), assuming that the contribution from the spectral continuum is negligible (and we do measure a very low continuum of less than $1 \%$ for the whole region). This approach is also justified by the fact that we use an HST $\mathrm{H} \alpha$ image (which includes continuum emission) for flux calibration (see below).

In order to flux calibrate the monochromatic map of 30 Doradus, we have used an archival HST image of this region, which was observed with the WFC3 and using the narrow-band filter F656N. We used the task PнOT in IRAF ${ }^{4}$ to measure the instrumental fluxes of 31 regions located across the field (which were located inside the same field of view of the SAM-FP $\mathrm{H} \alpha$ image), inside an aperture of 3 arsec radius. These instrumental values were converted into physical fluxes by using PHOTOFLAM (which is the 
so-called inverse sensitivity), $\mathrm{F}=1.78462 \times 10^{-17} \mathrm{erg}^{-1} \mathrm{~cm}^{-2}$ $\mathrm{s}^{-1} \AA^{-1}$ and the FWHM of the filter of $13.9 \AA$. Details can be found in the website of the Space Telescope Science Institute $^{5}$. Finally, each measurement was divided by the area within which the flux was estimated. For the SAM-FP 2D collapsed image, we obtained the number of electrons per second for the same regions defined for the HST image, inside the same apertures, using the same procedure (here we use the gain and exposure times listed in Table 1 to transform counts in electrons/s). In Figure 4 we show the result of this analysis, where a dashed-dotted line represents a linear fit to the data, with a slope of $1.32 \times 10^{-15} \mathrm{erg} \mathrm{cm}^{-2}$ electrons $^{-1}$ (we note that this value is consistent with the slope derived by forcing the zero point to zero, $1.38 \times 10^{-15}$ erg $\mathrm{cm}^{-2}$ electrons ${ }^{-1}$ ). Then the SAM-FP 2D image, i.e., the monochromatic image, was calibrated by using this coefficient (as shown by the scale on the right side of the top panel of Figure 3).

Most of the features visible in the monochromatic map of 30 Doradus have already been studied in the literature. For instance, the bright Northeast and a portion of the West filaments described as ionisation fronts by Rubio et al. (1998) and Pellegrini et al. (2010) are clearly outlined. Many dark clouds are easily seen in contrast against the bright nebula, for example the "stapler-shaped" dark cloud (Walborn, Barbá \& Sewilo 2013). Another structure seen is the dusty main cavity or Christmas Tree, which was detected by previous studies (e.g. Chu \& Kennicutt 1994, De Marchi \& Panagia 2014).

\subsection{A brief description of the kinematics of the observed region}

In the middle and bottom panels of Figure 3 we show the velocity field and the velocity dispersion map for the central region of 30 Doradus. Single Gaussian fits were used for obtaining these maps, regardless the number of line components. In the velocity field the fit traces the velocity peak at each pixel, which gives a general view of the kinematics of the nebula. In the case of the velocity dispersion map, the fit to a single Gaussian is very useful, even when multiple kinematic components are present, given that the width of the line allows the potential detection of regions with multiple kinematic components. The velocity dispersion map was corrected by instrumental and thermal widths, $\sigma_{\text {inst }}=11.3$ $\mathrm{km} \mathrm{s}^{-1}, \sigma_{t h}=9.1 \mathrm{~km} \mathrm{~s}^{-1}$, respectively. The latter value was estimated by assuming an electronic temperature of $\mathrm{T}_{e}=10^{4}$ $\mathrm{K}$ in the expression $\sigma_{t h}=\left(\mathrm{k} \mathrm{T}_{e} / \mathrm{m}_{H}\right)^{1 / 2}$.

One of the most intriguing features of the velocity field of 30 Doradus (middle panel of Figure 3 ) is the central region of the main cavity, or Christmas Tree (R.A $\sim 05 \mathrm{~h} 38 \mathrm{~m} 50 \mathrm{~s}$, Dec $\sim-69 \mathrm{~d} 05 \mathrm{~m} 52 \mathrm{~s})$. Around this location there is an abrupt change in the values of radial velocities, of $\simeq 40 \mathrm{~km} \mathrm{~s}^{-1}$, suggesting the existence of a complex kinematic structure. This map shows that the kinematics in 30 Doradus changes in small spatial scales. The structure of the velocity field poorly

5 http://www.stsci.edu/hst/wfc3/phot_zp_lbn and http://www.stsci.edu/hst/wfc3/documents/ISRs/2003/ WFC3-2003-02.pdf respectively. correlates with the $\mathrm{H} \alpha$ distribution, in contrast with the velocity dispersion map, which strongly correlates with it. In the velocity field, we observe a global velocity gradient of $\simeq$ $80 \mathrm{~km} \mathrm{~s}^{-1}$ from Southeast to North and Southeast to West. In addition, a component which either mimics or is a real continuous velocity component is observed from Southeast to Northwest $\left(\right.$ at $\left.\simeq 295 \pm 10 \mathrm{~km} \mathrm{~s}^{-1}\right)$.

Inspecting the bottom panel of Figure 3, we note that regions having the largest values of $\sigma$ are those for which two or more emission line profiles are superimposed at different velocities. This map is then a useful tool to search for expanding structures. We find that most of the high surface brightness regions (brighter than $5 \times 10^{-15} \mathrm{erg} \mathrm{s}^{-1}$ $\left.\mathrm{cm}^{-2}\right)$ display narrow profiles $\left(\sigma \sim 20-30 \mathrm{~km} \mathrm{~s}^{-1}\right)$ while lowintensity regions display broad multiple profiles $(70-80 \mathrm{~km}$ $\mathrm{s}^{-1}$ ), which arise from unresolved emission line components. In some cases the emission line profiles are clearly resolved in several peaks, specially in the region of the Christmas Tree. This area displays abrupt changes in the number of components, intensity and radial velocities of the ionised gas, which could be a result of the strong extinction along the line of sight.

In order to better display the kinematics of the inner region of the 30 Doradus Nebula, in Figure 5 we show the more relevant (in terms of flux) channel maps of the $\mathrm{H} \alpha$ data cube (channels 10 to $29,149.0$ to $392.2 \mathrm{~km} \mathrm{~s}^{-1}$ ). Starting from channel $16\left(225.8 \mathrm{~km} \mathrm{~s}^{-1}\right)$ we can see some emission associated with blue shifted gas. Between channels 18 (251.4 $\left.\mathrm{km} \mathrm{s}^{-1}\right)$ and $20\left(277.0 \mathrm{~km} \mathrm{~s}^{-1}\right)$ we have the peak of the emission. The cavity or Christmas Tree clearly appears in several channels. This cavity progressively disappears in channels 24 to $26\left(328.2 \mathrm{~km} \mathrm{~s}^{-1}\right.$ to $\left.353.8 \mathrm{~km} \mathrm{~s}^{-1}\right)$.

Perhaps the most striking result as we inspect the cube of 30 Doradus is the diversity of profiles on the smallest scales. This is exemplified in Figure 6 for an area of $9 \times 9$ arcsec. Here, each square corresponds to a profile binned $5 \times$ 5 pixel $^{2}$, i.e, over a $0.9 \times 0.9 \operatorname{arcsec}^{2}$ area (which corresponds to $0.23 \times 0.23 \mathrm{pc}^{2}$ ). The profiles change abruptly between neighbouring boxes, with components appearing and disappearing in scales of a fraction of a parsec (down to the spatial resolution of $0.15 \mathrm{pc}$ ).

A detailed analysis of the kinematics of the whole 2 ' $\times 2$ ' field, using tools such as the 3D spatio-kinematic code SHAPE (Steffen \& López 2006) to disentangle the 3-D geometry and kinematic structure of 30 Doradus, is deferred to a future paper.

\subsection{Identification of a new bubble in the centre of 30 Doradus}

The good spatial coverage and resolution of SAM-FP together with the reasonably good spectral resolution allows identification of new small-scale kinematic structures, which can not be easily identified in previous sparse multi-fiber data or in long-slit studies, where the positioning of the fibers and slits can bias the detection of such structures. Given that the aim of this paper is to show the capabilities of SAM-FP, here we show an example of an expanding structure, which appears when inspecting the $\mathrm{H} \alpha$ data cube. From the channel maps shown in Figure 5, one can see the presence of an expanding bubble identified here for the first time in the lower right of channels 20 to $25\left(277.0 \mathrm{~km} \mathrm{~s}^{-1}\right.$ to 

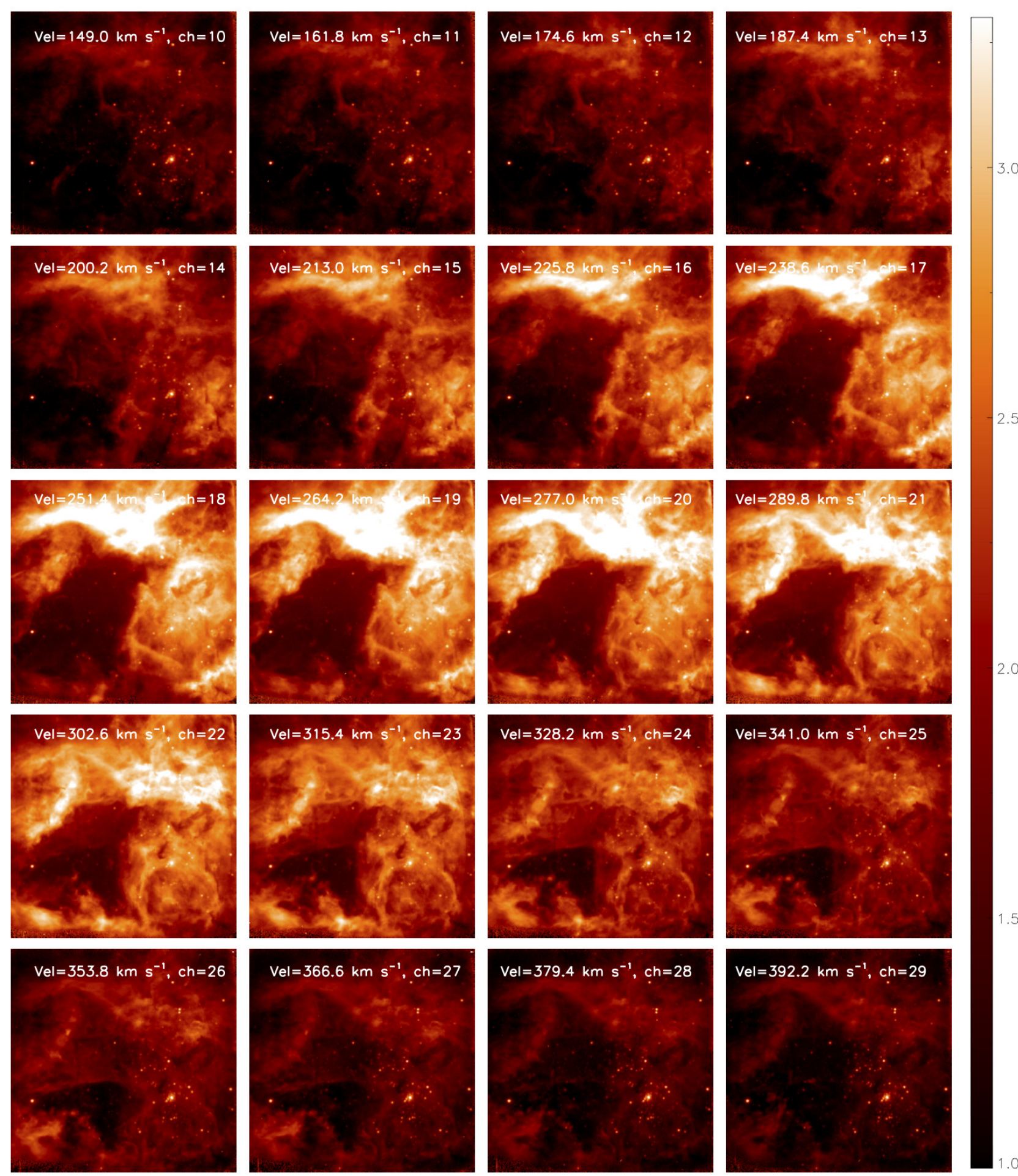

Figure 5. Channel maps for the $\mathrm{H} \alpha$ data cube of 30 Doradus. We show here the 20 channels (of the 40 channels observed) with most emission. The Northeastern filament is the bright emission on the upper parts of the frames (seen in its brightest emission from channel 16 to channel 21). The Christmas Tree can be seen, in most channels, as the dark feature in the Southeast of the frames. The mean velocity of the channel and the channel number are shown at the top of each stamp. 

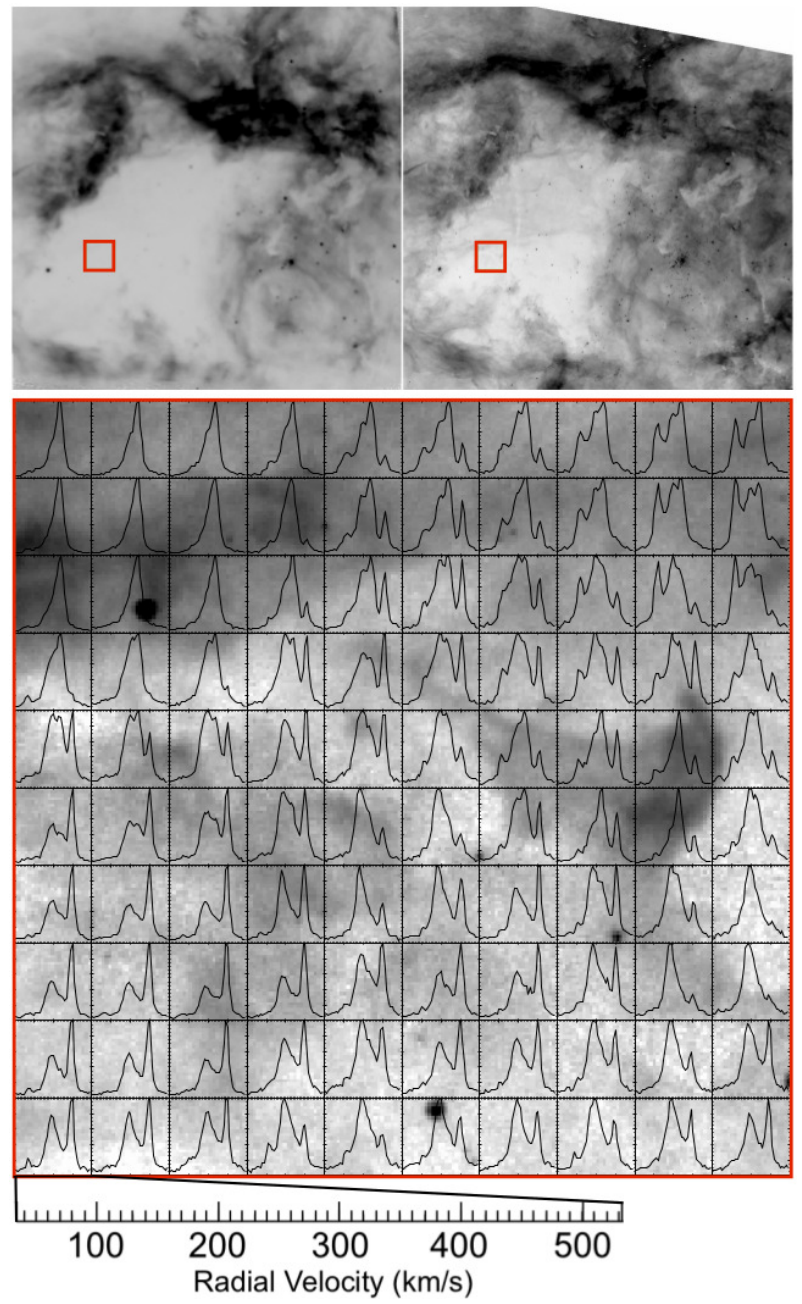

Figure 6. Profiles of one selected area of 30 Doradus. Top left pannel: $\mathrm{H} \alpha$ image extracted from channel 21 of the Fabry-Perot data cube (see Figure 5 for a color version of this channel). The red square indicates the region whose profiles are shown in the lower panel. Top right panel: HST H $\alpha$ image of the same region displayed on the top left. Bottom panel: $\mathrm{H} \alpha$ profiles over a $5 \times$ 5 array of binned pixels. Each "pixel" is $0.9 \operatorname{arcsec}(0.23 \mathrm{pc})$ on a side. The purpose of this Figure is to show the diversity of profiles in a small area of only $9 \times 9 \operatorname{arcsec}^{2}\left(0.23 \times 0.23 \mathrm{pc}^{2}\right)$. This diversity is typical of the whole area observed. Note that the profiles can be easily traced from one pixel to the next. The velocity scale is shown on the lower left, in one case, and it is the same for all profiles.

$341.0 \mathrm{~km} \mathrm{~s}^{-1}$ ). The presence of this bubble is not obvious in the velocity field of 30 Doradus (middle panel of Figure 3), but it can be identified as a region of broader profiles (i.e. larger widths - when using a single Gaussian fit) in the velocity dispersion map (bottom panel of the same Figure). We attempt to show this bubble more clearly in the top panel of Figure 7, where a slice of the Fabry-Perot data cube centred at velocity $315.4 \mathrm{~km} \mathrm{~s}^{-1}$ (channel 23) is displayed. The white circle indicates the location of the expanding bubble centred at R.A 05h 38m 42s, Dec -69d 06m 23s.

Torres-Flores et al. (2013) identified a large bubble that encompasses this region (their region 8) and also includes the central cluster R136. However, they had a poor spatial coverage of one spectrum each 20 arcsec. With the present dataset we can more precisely identify the location and size of this expanding bubble. In Figure 7 (top panel) it is possible to see arc-like features, which delineate the structure, approximately following the contours of the white circle. The kinematic signature of this source is shown in the bottom panel of Figure 7, which is the result of adding all the $\mathrm{H} \alpha$ profiles that are enclosed inside the white circle (top panel). In this plot, we can see a double-peaked profile, which is typically associated with expanding structures. In order to quantify the expansion velocity of this structure, we have fitted two Gaussians to the profile (plus a low-intensity continuum emission). This procedure allows us to derive the radial velocity of both components. Under the assumption that these components are associated with the approaching and receding sides of the expanding bubble, the expansion velocity of the bubble can be estimated as $\mathrm{V}_{\text {expansion }}=$ $\left(\mathrm{V}_{\text {receding }}-\mathrm{V}_{\text {approaching }}\right) / 2$. This exercise yields a velocity of $\mathrm{V}_{\text {expansion }}=29 \pm 4 \mathrm{~km} \mathrm{~s}^{-1}$. The radial velocity of the barycentre of the bubble, $\left(\mathrm{V}_{\text {receding }}+\mathrm{V}_{\text {approaching }}\right) / 2$, is $273 \mathrm{~km} \mathrm{~s}^{-1}$. The error of $4 \mathrm{~km} \mathrm{~s}^{-1}$ is obtained from the velocity difference between the barycentre measured on the observed doublepeak profiles and the barycentre derived from the sum of the two fitted Gaussians. Considering that the bubble has a projected radius of $22 \operatorname{arcsecs}$ or $5.6 \mathrm{pc}$, we can speculate that this structure has been expanding for a period of $\sim 180,000$ years, assuming a constant and uniform expansion.

The ionising source (or sources) associated with this expanding bubble cannot be easily identified using FP data alone, given that the continuum emission is poorly sampled in the data-cube. Inspecting the population of massive stars in the central $10 \times 10 \operatorname{arcsec}^{2}$ (or $2.5 \times 2.5 \mathrm{pc}^{2}$ box) of the proposed bubble, we have two remarkable stars, indicated in Figure 7: the blue supergiant Melnick 38 (VFTS 525, B0 Ia, Walborn et al. 2014) and the very massive spectroscopic binary VFTS 512 (O2 V-III, Walborn et al. 2014). These stars are located 2.7 arcsec and 2.4 arcsec from the centre of the bubble, respectively, and they have different reasons to be the source of the expanding nebula. In the case of VFTS 512, we could be witnessing the birth of a pristine young bubble produced by a powerful wind and UV-radiation of a very young massive star. In the case of Melnick 38, the origin of the nebula could be related to advanced evolutionary stage of a blue supergiant. Walborn et al. (1997) described that in 30 Doradus at least five distinct populations of massive stars are coeval, from an embedded population of massive stars (see also Rubio et al. 1998, Walborn, Barbá \& Sewilo 2013), the Orion Nebula-like population, to a more evolved sparse population, composed by blue and red supergiants, the Sco-Cen OB association-like population. Nazé et al. (2001) searched for pristine bubbles in two very young HII regions of the LMC, N11 and N180. They discovered a very small bubble around the multiple star PGMW 3120, with a composed spectral type of $\mathrm{O} 5.5 \mathrm{~V}((\mathrm{f}))$, which is described as a very young asymmetric bubble by Barbá et al. (2003).

The new expanding bubble found in this work is located close to the star cluster R136 (its centre is 5.6 arcsec South of R136, in projection). Although the mechanical feedback of R136 stars could disrupt any small-scale kinematic structure located in its environment, this does not seem to be the case here, given the apparently smooth shape of the bubble contours and its distinct kinematics. Probably this bubble 

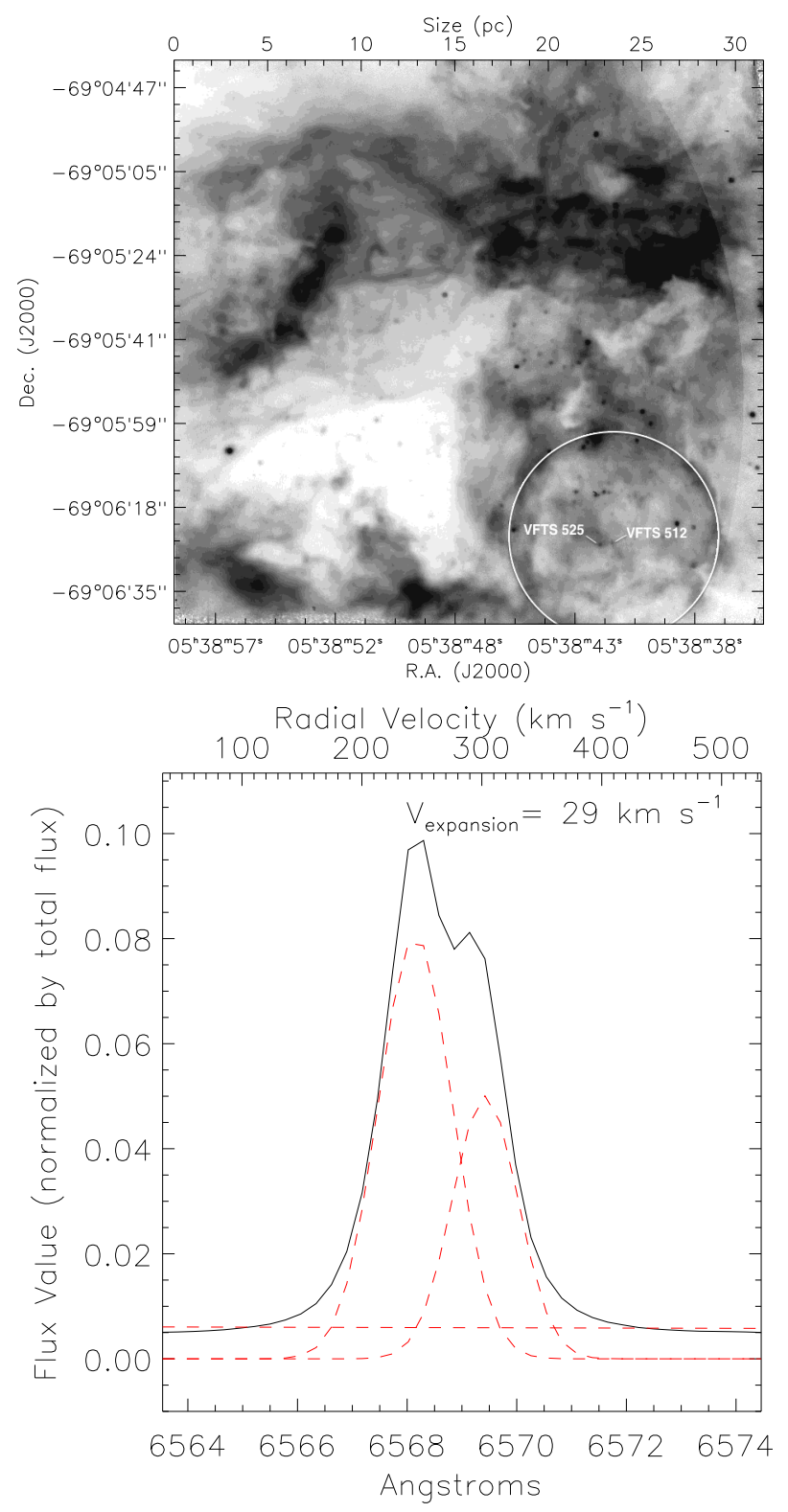

Figure 7. Top panel: channel $23\left(315.4 \mathrm{~km} \mathrm{~s}^{-1}\right)$ of the 30 Dor data cube, where the white circle indicates the location of the expanding bubble. The blue supergiant Melnick 38 and the very massive spectroscopic binary VFTS 512 are indicated. Lower panel: integrated $\mathrm{H} \alpha$ profile for the region contained within the white circle shown in the top panel. Red dashed lines represent two Gaussian components plus a continuum component, which can be identified as a horizontal line, with a value of $\sim 0.006$. On the top right we show the value of the expansion velocity derived from the average of the velocities of the two peaks (receding and approaching sides).

is not located immediately next to R136 and the spatial coincidence we see is just a chance alignment, in the line of sight. Projection effects should be taken into account in the identification and analysis of bubbles.

\subsection{The integrated $\mathrm{H} \alpha$ profile}

A few studies to date have suggested that the integrated $\mathrm{H} \alpha$ profile of 30 Doradus is composed by a broad component, due to a low-intensity emission, superimposed on a narrow, high-intensity component. This may be caused by the sum of expanding structures or may be the result of the superposition of a number of narrow, low-intensity components with different radial velocities. We expect the broad component to be quite faint and therefore a detailed and careful analysis and modelling of the line profiles, taking into account the underlying intrinsic Fabry-Perot Airy function, needs to be done. While this problem is outside the scope of the present paper, it is still interesting to measure the width of the integrated emission line of the whole region observed, to compare with that measured in the previous work of Torres-Flores et al. (2013). For this, we stacked all profiles of the 30 Doradus cube (a $2 \times 2 \operatorname{arcmin}^{2}$ region). We then fit a single Gaussian to the integrated $\mathrm{H} \alpha$ profile, which is shown in Figure 8. The black continuous line in this Figure corresponds to the integrated observed profile, while the red dashed line is the modeled single Gaussian (which was obtained by using the code PAN in IDL). We corrected the profile for instrumental and thermal broadening, in a similar way done for the velocity dispersion map and we then computed its dispersion to be $\sigma \sim 34 \pm 1 \mathrm{~km} \mathrm{~s}^{-1}$. The error of $1 \mathrm{~km} \mathrm{~s}^{-1}$ is obtained from the velocity difference between the barycentres of the observed profile and of the Gaussian fit. The value obtained here for the dispersion is higher than the one found by Torres-Flores et al. (2013) of $\sigma=26.5 \mathrm{~km}$ $\mathrm{s}^{-1}$, but that was for a much larger region of $10^{\prime} \times 10^{\prime}$ centred on R136. These two values are consistent, given the uncertainties due to different resolutions and different sizes of the regions used in the determination.

In a forthcoming publication (Torres-Flores et al. in preparation) we will take into account the shape of the Fabry Perot profile (Airy Function) to correctly investigate the presence of wings in the integrated $\mathrm{H} \alpha$ profiles, after bringing all different radial velocity profiles to a common zero point velocity.

\subsection{Visualizing the $\mathrm{H} \alpha$ data cube in 3D}

$3 \mathrm{D}$ visualization of the $\mathrm{H} \alpha$ Fabry-Perot data cube of 30 Doradus can help us understand the structure and kinematics of the nebula.

Figure 9 shows one such 3D representation, where right ascension, declination and velocity (wavelength) of 30 Doradus are plotted. In order to highlight small scale kinematic features, the wavelength axis has been resampled to 350 channels (instead of the original 40 channels). The panels of Figure 9 were produced with GLnemo2 (Lambert 2016), an interactive $3 \mathrm{D}$ program that allows visualising the data cube in different directions. These are indicated by arrows in the right-hand corner of each panel, where the red, green and blue arrows correspond to right ascension, declination and velocity (wavelength), respectively. Depending on the angle along which the data cube is seen, we can obtain from a face-on (0-degree view) image to a position velocity map (90-degree view). Figure 9 shows a $3 \mathrm{D}$ view with rotation around the declination axis, with a frontal view, then a 45degree, a 70-degree and a 90-degree view of the system. Re- 


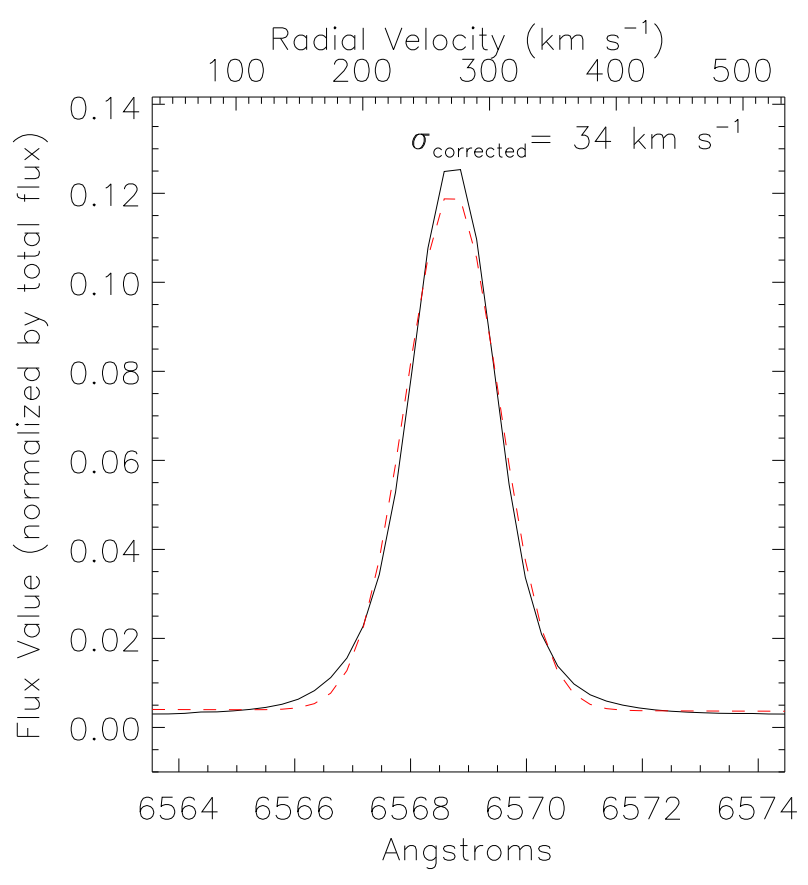

Figure 8. Integrated $\mathrm{H} \alpha$ profile of 30 Doradus, derived from the whole data cube (black continuous line). The red dashed line corresponds to a single Gaussian fit to the observed profile. On the top left corner we list the value of the width $(\sigma)$ of the integrated profile, which has been corrected by instrumental and thermal widths.

gions of greatest intensity are shown in red while blue regions are those with lowest intensity and the straight lines are the stars, which help constructing the $3 \mathrm{D}$ view.

The contours of the cavity or Christmas Tree are very well defined in the first three panels of Figure 9 (all except the 90-degree panel), given the contiguous coverage of emission over the field. In the top, a frontal view is shown, where several low-luminosity structures can be seen within the cavity, which are thought to be filaments being ejected in the line of sight. They were seen by both Melnick et al. (1999) and Chu \& Kennicutt (1994) in 2D images and long-slit spectra as numerous gas blobs moving with more than 100 $\mathrm{km} \mathrm{s}^{-1}$ with respect to the system. This suggested to these authors that this is a region where expanding wind-driven shells undergo break-out. These kinds of structures were also identified by Redman et al. (2003) in another region of 30 Doradus. In this sense, a detailed analysis of $\mathrm{H} \alpha$ SAM-FP data will be extremely valuable in order to determine the main mechanism producing these discrete knots.

The different radial velocities across the nebula shown in the 90-degree cut (the position-velocity diagram) also indicate the existence of several kinematic structures, possibly parts of one or more expanding shells.

Another remarkable feature of Figure 9 is the clear indication that the Northeast filament (Rubio et al. 1988, the filament that runs horizontally on the top part of the Figure) has a different velocity than the Eastern filamentary loop (also identified as ionisation front 2, or "if2", Pellegrini et al. 2010). If we measure the difference in velocity between the channel where the Northeast filament is brightest and that where the Eastern loop is brightest, we find $37 \pm 13 \mathrm{~km}$ $\mathrm{s}^{-1}$ (we consider the error to be \pm one channel, or $\sim 13 \mathrm{~km}$ $\left.\mathrm{s}^{-1}\right)$.

There is a clear velocity gradient of the Northeastern filament, with increasingly more negative velocities running from West to East. This can be clearly seen in the position velocity diagram (bottom panel). Note that in this figure the Northern part of the nebula, in projection, is clearly bent to the right, indicating blue shift with respect to the bulk of the gas. As described by Westmoquette et al. (2010), when a gas cloud hits a molecular cloud, it flows around it and we see only the components that are facing towards us (blueshift), given that the components that go behind the cloud are screened by the cloud itself. This process may be happening in the Northeastern filament, with gas clouds coming from the Southwest and hitting a large molecular cloud detected by several previous works (e.g. Rubio et al. 1988 and references therein). This process which here appears to happen in a scale of $25 \mathrm{pc}$ or larger scales has also been suggested by Westmoquette et al. (2010) to occur around the Eastern pillar of NGC 6357 in a much smaller scale.

The preliminary analysis described above shows clearly that the detailed kinematic map derived for complexes and filaments of 30 Doradus may allow identification of several kinematically independent structures.

\section{SUMMARY AND CONCLUSIONS}

We present the first results obtained with a Fabry-Perot mounted inside SAM, a ground-layer adaptive optics instrument using laser, on SOAR. The data cubes had a spectral resolution of $R \simeq 11200$ at $\mathrm{H} \alpha$ and a FWHM of $\simeq 0.6$ arcsec. This configuration provides a unique tool to study the kinematics of a variety of astronomical sources, such as normal and interacting galaxies, merging objects and Galactic objects such as $\mathrm{HH}$ objects, planetary nebulae, and Giant HiI regions, with good spatial and spectral resolution. Note, however, that some targets, which have velocity ranges larger than the FSR of $492 \mathrm{~km} / \mathrm{s}$, are not appropriate for SAM-FP when used with the Fabry Perot device described in this paper. FPs with larger FSR must be used, for example, to observe some planetary nebulae fragments, novae shells and supernova remnants, which can exhibit much faster velocities (e.g. the knots of MyCn 18, Redman et al. 2000, or nova GK Pe, Liimets et al. 2012) that will not be captured by this velocity range. Moreover, objects with broad lines $(>8 \AA)$, such as active galactic nuclei, have to be observed with an FP with a larger FSR. In a future paper we will describe a second Fabry Perot that may be useful in such cases.

An integral part of this paper is the material presented in the appendices, which provide recommendations on how to use Fabry-Perot instruments and how to understand the observations, providing all formulae involved. A few examples of topics discussed in the appendices are given in the following. A long standing confusion of observers about the difference between the scanning sampling and the resolution is clearly explained in appendix A, while the phase-shift effect, which is a physical effect linked to the coating of the etalons is described in appendix B. We clarify when and why the observer may choose to scan a full FSR or a fraction of 
it or more than a FSR and we give some practical information for performing observations, such as for example, we recommend that the observer obtain a new observation in a position free of an object, for sky subtraction, when the object is larger than the field of view. Without this knowledge, no proper observations can be done or the observed data will not be scientifically useful. Thus, the appendices are meant to be useful not only for users of SAM-FP but users of Fabry Perot instruments in general.

In order to highlight the capabilities of SAM-FP, we observed the region of 30 Doradus, for which there are previously published kinematic data. We show an overall kinematic description of the central part of the 30 Doradus nebula in the LMC. The current configuration allows us to obtain kinematic information on scales of $0.15 \mathrm{pc}$, which can be improved under better seeing conditions.

Our main conclusions on the study of 30 Doradus are:

Comparing our Fabry-Perot data with previously taken VLT/FLAMES observations, we find good overall agreement in the regions of overlap, but the SAM-FP data cubes have a filling factor 360 times higher than the comparison data cubes.

The Fabry-Perot dataset has great advantages over other multi-fiber or long-slit data in the search for bubbles in nebulae in general and in 30 Doradus in particular, given its much improved spatial coverage. Analysis of the FabryPerot data reveal a new bubble at a projected distance of 22 arcsec South of R136 with an expansion velocity of $29 \pm$ $4 \mathrm{~km} \mathrm{~s}^{-1}$.

The Eastern filamentary loop of 30 Doradus is redshifted by $\sim 37 \pm 13 \mathrm{~km} \mathrm{~s}^{-1}$ with respect to the Northeastern filament indicating that these complexes are kinematically independent.

A velocity gradient in the Northeastern filament increases to more negative velocities running from West to East. This suggests the presence of gas flowing around the molecular clouds, where we only see the component facing towards us. A similar mechanism was invoked in smaller scales around the Eastern pillar of NGC 6357 (Westmoquette et al. 2010)

Small high-velocity clouds are seen inside the cavity known as Christmas Tree. These are also the same kinds of phenomena reported by Redman et al. (2003), where the shell is fragmented by perturbations (such as small molecular clouds) in the medium.

In a future paper we will focus on the analysis of the whole data cube and we will specifically study the ionised gas structure around the massive stars, the relationship between their spectral types, evolutionary stage and the kinematics of the $\mathrm{H} \alpha$ profiles associated to them. The aim is to identify a number of pristine HiI regions and small bubbles around massive stars and to evaluate the evolutionary scenario of the bubbles. In this context, we will attempt to describe the elusive kinematics of the small bubbles in the caldera of a giant HiI region (only possible with the data taken in this work). Finally, this new instrument opens the possibility of exploring the synergy between HST and FP data given that the wealth of details seen in HST images of nearby giant His regions can now be matched by similar quality kinematic data taken from the ground, with SAM-FP and laser correction.
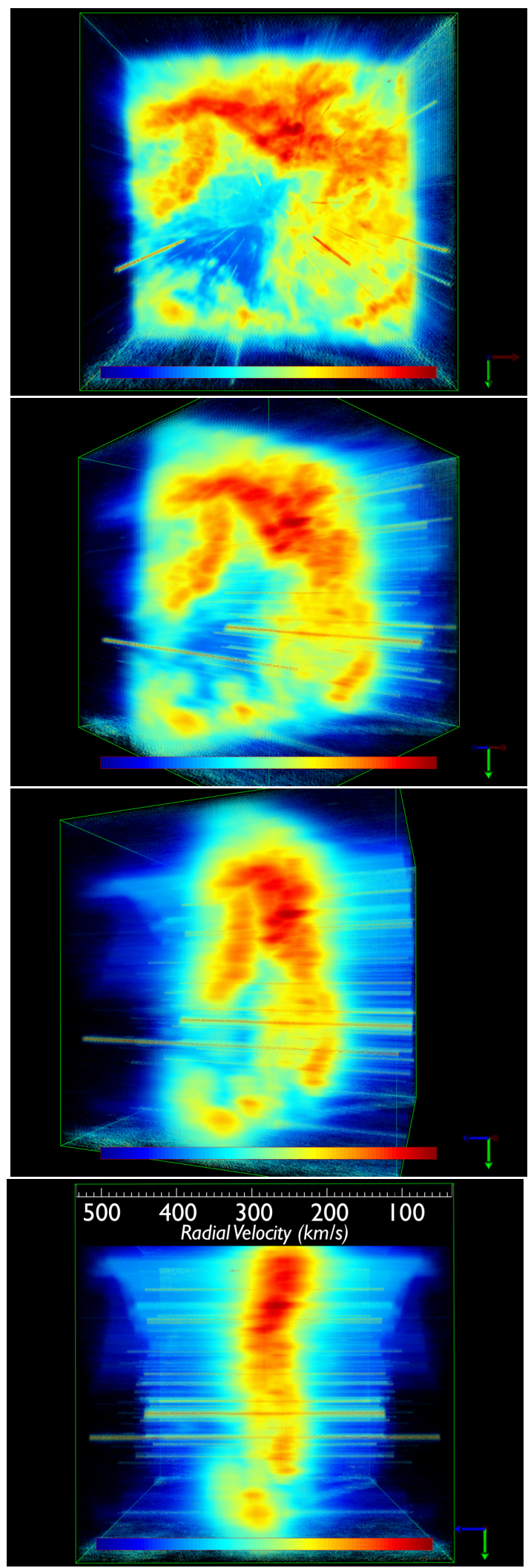

Figure 9. $3 \mathrm{D}$ view of the H $\alpha$ Fabry-Perot data cube of the central part of 30 Doradus with rotation around the declination axis. Top: frontal view of 30 Doradus; second from top to bottom: 45-degree view; third from top to bottom: 70-degree view; bottom: 90-degree view. The color bar shows a relative intensity scale. A velocity scale is given at the top of the lowest panel. Continuous lines are stars in the field. This Figure was prepared with the software GLnemo2 to facilitate visualisation.

MNRAS 000, 1-?? (2016) 


\section{ACKNOWLEDGMENTS}

We warmly thank Marco Bonatti for writing the control software for scanning the Fabry-Perot inside SAM. We thank Brian Chinn for designing the mechanical mount for including the Fabry-Perot in SAM. We thank Dr. Warrick Couch, Dr. Matthew Colless and the AAO for kindly loaning us the Fabry-Perot instrument used for this work. We thank Dr. Andrei Tokovinin for extensive help with the observing runs and for very useful comments on the manuscript. We are grateful to Dr. Tiago Ribeiro for taking the image of 30 Doradus used in Figure 1 with the T80-South telescope and to the T80-South team for allowing us to use the image in this paper. We greatly appreciated the help by Luciano Fraga, who agreed to exchange one of his nights with ours, allowing tests and science observations to be done with SAM-FP. We thank Dr. Benoît Epinat for fruitful discussions on Fabry-Perots, Mohamed Belhadi and Amandine Caillat for measuring the passbands of the interference filters in several configurations and Jean-Charles Lambert for facilitating the use of GLnemo2. We are greatly in debt to Dr. Keith Taylor, Rene Laporte and INPE, for continuous support over the last many years to the BTFI and SAM-FP teams. We thank Dr. Christof Iserlohe, for writing the routine fluxer, which was used in this work. We thank an anonymous referee who made very useful suggestions to the original version of the manuscript. CMdO and DA thank FAPESP and CNPq for financial support through grants 2009/54202-8 and 2014/07586-2. ST-F acknowledges financial support from the "Direccion de InvestigaciÂşn y Desarrollo de la ULS", through a project "DIULS Regular", under contract PR16143. RB acknowledges financial support from FONDECYT, through Project 1140076. ST-F and CMdO acknowledge financial support of CONI$\mathrm{CYT}+\mathrm{PAI} /$ Atracci $\tilde{A} s ̧ n$ de Capital Humano Avanzado del Extranjero, Folio NÂž PAI80160082. CMdO and PA thank the support of the USP-Cofecub international collaboration program, which made this project possible. In particular the BTFI instrument described in Mendes de Oliveira et al. (2013) and the papers Torres-Flores et al. (2013b, 2014) and Alfaro-Cuello et al. (2015) were possible thanks to the research exchange program supported by USP-Cofecub, to which we greatly acknowledge. Based on observations obtained at the Southern Astrophysical Research (SOAR) telescope, which is a joint project of the Ministerio da Ciência, Tecnologia, e Inovação (MCTI) da República Federativa do Brasil, the U.S.A. National Optical Astronomy Observatory (NOAO), the University of North Carolina at Chapel Hill (UNC), and Michigan State University (MSU).

\section{REFERENCES}

Alfaro-Cuello, M., Torres-Flores, S., Carrasco, E. R., Mendes de Oliveira, C., de Mello, D. F., Amram, P. 2015, MNRAS, 453, 1355

Amram, P., Marcelin, M., Boulesteix, J., Le Coarer, E. 1989, A\&AS, 81, 59

Atherton, P.D., Reay, N.K., Ring, J., \& Hicks, T.R. 1981, Tunable Fabry-Perot Filters, Optical Engineering, Vol. 20, No. 6, pp. 806-814

Atherton, P.D., Taylor, K., David Pike, C. et al. 1982, MNRAS, 201,661
Barbá, R. H., Rubio, M., Roth, M. R. and García, J. 2003, AJ, 125,1940

Bland, J., Taylor, K., Atherton, P.D. 1987, MNRAS, 228, 595

Chu, Y. H., Kennicutt, Jr. R. C. 1994, ApJ, 425, 720

De Marchi, G., Panagia, N. 2014, MNRAS, 445, 93

Dimeo, R., PAN User Guide, available online at ftp://ftp.ncnr.nist.gov/pub/staff/dimeo/pandoc.pdf

Fraga, L., Kunder, A., \& Tokovinin, A. 2013, AJ, 145, 165

Lambert, J.C., CeSAM, Laboratoire d'Astrophysique de Marseille, Internal note, 2016

Liimets, T., Corradi, R. L. M., Santander-Garciía, M., Villaver, E., Rodríguez-Gil, P., Verro, K., Kolka, I. 2012, ApJ, 761, 34

Melnick, Jorge, Tenorio-Tagle, Guillermo, Terlevich, Roberto 1999, MNRAS, 302, 677

Mendes de Oliveira, C., Taylor, K., Quint, B., et al. 2013, PASP, 125,396

Nazé, Y., Chu, Y.-H., Points, S. D., Danforth, C. W., Rosado, M. and Chen, C.-H. R. 2001, AJ, 122, 921

Pellegrini, E.W., Baldwin, J. A. Ferland, G. J. 2010, ApJS, 191, 160

Redman, M. P., O'Connor, J. A., Holloway, A. J., Bryce, M., Meaburn, J. 2000, MNRAS, 312, L23

Redman, M.P., Al-Mostafa, Z. A., Meaburn, J., Bryce, M. 2003, MNRAS, 344, 741

Rúbio, Mônica, Barbá, Rodolfo H., Walborn, Nolan R., Probst, Ronald G., García, Jorge, Roth, Miguel R. 1998, AJ, 116, 1708

Steffen, W. \& López, J.A. 2006, Rev. Mex. Astron. Astrofis., 42, 99

Tokovinin, A., Tighe, R., Schurter, P., et al. 2008, Proc. SPIE, 7015, article id. $70154 \mathrm{C}$

Tokovinin, A., Tighe, R., Schurter, P., et al. 2010, Proc. SPIE, 7736, article id. $77363 \mathrm{~L}$

Tokovinin, A., Tighe, R., Schurter, P., et al. 2012, Proc. SPIE, 8447, article id. $84474 \mathrm{H}$

Torres-Flores, S., Barbá, R., Maíz Apellániz, J., Rúbio, M., Bosch, G., et al. 2013, A\&A, 550, A60

Torres-Flores, S., Mendes de Oliveira, C., Plana, H., Amram, P., Epinat, B. 2013, MNRAS, 432, 3085

Torres-Flores, S., Scarano, S., Mendes de Oliveira, C., de Mello, D. F., Amram, P., Plana, H. 2014, MNRAS, 438, 1894

Veilleux, S., Weiner, B. J., Rupke, D. S. N., McDonald, M., Birk, C., Bland-Hawthorn, J., Dressler, A., Hare, T., Osip, D., Pietraszewski, C., Vogel, S. N. 2010, AJ, 139, 145

Walborn, Nolan R., Barbá, Rodolfo H., Sewilo, Marta M. 2013, AJ, 145,98

Walborn, N. R. and Blades, J. C. 1997, 112, 457

Walborn, N. R., Sana, H., Simón-Díaz, S. et al. 2014, A\&A, 564, A40

Westmoquette, M.S., Slavin, J.D., Smith, L.J., Gallagher, J.S., IIII 2010, MNRAS, 402, 152

\section{APPENDIX A: WHAT IS NEEDED FOR CARRYING OUT FABRY-PEROT OBSERVATIONS}

This appendix is written as a recipe. First we describe how to select the calibration lamp and the interference filter. Then we give the list of parameters that need to be provided/computed to obtain Fabry-Perot observations. Finally some recommendations on how to perform the observations are given. Section 2.2 of the main text and Appendix B explain why these steps should be followed. 


\section{A1 How to choose the right calibration lamp?}

The calibration lamp must contain at least one emission line passing through an available interference filter. In order to minimize additional phase-shift effects, the interference filter for the calibration should ideally be the target interference filter (defined here as the interference filter suitable for the observation of the on-sky target). However, often no narrow emission lines are available within the target interference filter, so the observer should select a calibration-lamp narrow emission line as close as possible to the observed emission line $(\mathrm{H} \alpha$ in our case, redshifted due to the velocity of the target). Around $\mathrm{H} \alpha$, the $6598.99 \AA$ Neon emission line is suitable for nearby objects and a filter, centred, e.g. on 6600 $\AA$, with $20 \AA$ width, can then be used. Further explanations are given in Section B1.

\section{A2 How to choose the right interference filter?}

Optical Fabry-Perot instruments are typically optimised to work in the blue or in the red, depending on the plates' coating. The observer should further narrow down the spectral range of interest by selecting the target interference filter. The FWHM of the target interference filter should match and in practice should be slightly larger than one FSR. The FSR (formula 2) depends on the wavelength and on the interference order and it is typically $11 \AA$ for the Fabry Perot described in this paper. Thus the target interference filter could have a FWHM between 12 and $25 \AA$ (note that if the filter width is too large, then different orders of a given wavelength will appear in different parts of the data cube causing possible confusion due to line overlapping). The right interference filters have to be chosen for both the calibration and object observations. Further explanations are given in section B2.

\section{A3 Which input parameters are needed for performing FP observations?}

\section{A3.1 How to compute the optimal number of steps?}

The computation of the optimal number of steps $\mathrm{n}$ needed to scan a FSR is described in this section. To satisfy the Nyquist-Shannon's sampling criteria, $\Delta e_{A}$ must be sampled at least on two channels. In order to minimize the number of scanning channels, the Airy function is then sampled with two channels only and thus the number of channels needed to scan a FSR is simply $n=(2 \times F)$, recalling that $\mathrm{F}$ is the effective finesse of the etalon. Given that $\mathrm{F}$ is a float number and $\mathrm{n}$ is an integer, then $n=(2 \times F)+1$. For instance the ICOS ET-65 etalon used for this work has an effective finesse $F \simeq 18.5$, thus $n=38$. The observer is nevertheless free to choose another number of steps $\mathrm{n}$ in order to undersample or oversample the scanning sequence but of course it will not change the resolution $R_{\lambda}$. This is indeed what we did, in the case of the observations of 30 Doradus, we gently oversampled the scanning of the effective finesse by $10 \%$ leading to $n=40$ scanning channels and to a scanning step of 12.8 $\mathrm{km} \mathrm{s}^{-1}$. The determination of the FSR and of the effective finesse are given in sections B3.2 and B3.3 respectively.

\section{A3.2 How to choose the scanning wavelength?}

The scanning wavelength for the object must correspond to its mean radial velocity. The scanning wavelength for the calibration must be the wavelength of the calibration line.

\section{A3.3 Which range to scan?}

The observer may chose to scan a fraction of the FSR, exactly the FSR or more than one FSR. The first option is not typically recommended for high-order Fabry-Perot instruments because the whole field of view will not be covered by the FSR. The third option could be selected if the observer needs to check the FSR during the reduction procedure. To optimize the observing time we recommend the second option, i.e., to scan exactly one FSR. Further technical details on the way to best define the scanning sequence is provided in section B3.4.

\section{A3.4 How to choose the observing time?}

The description below assumes that the Fabry Perot instrument uses a camera with a high-quality CCD detector, which is the case in most large observatories.

First of all, the observer has to determine the observing time per individual channel. This time is a compromise between a reasonable number for the total time spent on target at a given stable atmospheric condition and the optimization of the use of the detector (given its read noise characteristics). Cosmic ray hits also impose an upper limit to the exposure time per channel (to a maximum of about 5 minutes). As an example, for a scanning sequence with 40 steps, the exposure per channel should not be longer than 2 minutes, given that this leads to more than 80 minutes for the total cycle, which is already a significant time during which the atmospheric conditions may have changed (airmass, transparency and seeing). A shorter exposure time per channel is not advisable for low-surface brightness objects, given the typical readout noise of modern CCDs $(\sim 3$ electrons). In case the observer wants to increase the signalto-noise ratio of the observation, several cycles should be repeated. Much shorter exposure times per channel (say 130 seconds) are suitable for high surface brightness sources (e.g. bright planetary nebulae and bright HII regions).

\section{A3.5 How to choose the pixel binning?}

It is advisable to use binned pixels to match NyquistShannon's sampling criteria, avoiding high spatial oversampling. Binning will considerably increase the SNR per unit surface and this is mandatory for low-surface brightness objects, given the readout noise of the CCD and the typically short exposures used in Fabry-Perot observations.

\section{A4 Standard observing procedures}

\section{A4.1 About wavelength calibrations}

Once the scanning sequence is defined, the observer can use it for the wavelength calibration. We recommend that lamp calibration frames are taken during day time to check the Fabry-Perot set-up and the scanning procedure (in this case 
the scanning wavelength must correspond to zero velocity, i.e. rest wavelength). As explained in section 2.2 .2 , it is recommended that the observers do wavelength calibration before or after the target exposure with the telescope in the same position to match as much as possible the conditions of the observations.

\section{A4.2 About subtraction of the night sky-line emission}

If the target does not fill the whole field of view of the detector, the night sky line could be safely derived and subtracted during the reduction procedure (using the galaxy-free area to measure the sky lines). If the target does fill the whole field of view, two cases may be envisaged. First, if the spectral density is low (i.e. the average wavelength range of the target emission line per pixel is less than about one third of the FSR), the night sky line could be identified using medians during the reduction procedure. Second, if the spectral density is high, it is recommended to measure the sky from a new pointed observation on a target-free location, just beside the target. As the sky is expected to not have spatial structure, a short exposure should be enough (typically one tenth of the exposure time used for the target). After wavelength calibration, spectra from all pixels can then be summed up.

\section{A4.3 About flux calibrations and flat fields}

Flux calibrations for Fabry-Perot observations should be done using standard procedures like any other instrument, i.e. by observing calibration standards. We nevertheless recommend to use spatially extended calibrated emission line sources like planetary nebulae or isolated HII regions. A scanning sequence with the same parameters used for the target observations must be used (with the important difference that the scanning wavelength must be the wavelength of the calibration source, i.e., in the case of a planetary nebulae it should correspond to the radial velocity of the planetary nebula). Flat fields should be taken to correct the spectrum from pixel to pixel efficiency variations and the effect of the interference filter transmission curves. The Fabry-Perot should not be in the beam during the procedure of obtaining flat fields (so no scanning is necessary).

\section{APPENDIX B: WHAT IS NEEDED FOR UNDERSTANDING FABRY-PEROT OBSERVATIONS}

To complement the main text and Appendix A, this appendix gives the relevant explanations to understand FabryPerot observations.

\section{B1 Wavelength calibrations and additional phase-shift effect}

Before obtaining on-sky observations one must take a few reference calibration cubes for checking the Fabry Perot parameters and for subsequent wavelength calibration. This is usually done in day time, prior to the first night of observations. As described in Appendix A1, one must select an arc line (from one of the calibration lamps available at the telescope) which has a wavelength which is similar to that of the emission line to be observed.

Selecting a proper arc line to be used for wavelength calibration is a crucial step for any Fabry Perot observation due to the additional phase-shift problem explained below. In textbooks on Fabry-Perots, it is stated that the transmission curve of a Fabry-Perot is not a function of the wavelength within a given spectral band. However, in practice, the reflectivity of the coatings deposed onto the inner side of the two plates do depend on the wavelength. Each reflection, due to each dielectric coating, having complex transmission and reflection coefficient numbers, induces an additional phase-shift $\psi(\lambda)$, also called phase-lag, which depends on $\lambda$ in the range $[0,2 \pi]$. Then equation (1) becomes:

$\mathrm{p}=\frac{2 \mathrm{ne} \cos \theta}{\lambda}-\frac{2 \psi(\lambda)}{2 \pi}$

where $\mathrm{p}$ is now the effective interference order, $\mathrm{n}\left(\mathrm{T}=0^{\circ} \mathrm{C}, \mathrm{P}=1 \mathrm{~atm}\right) \simeq 1.0003$ is the index of the air layer between the two plates of the Fabry-Perot device, e the inner separation between the coated plates and $\theta$ the incidence angle. What happens is that the coatings, which consist of the superposition of multiple thin dielectric layers (typically 0.1-1 $\mu \mathrm{m}$ each), alternating transparent dielectric material with low refractive index (typically $\mathrm{n} \simeq$ 1.5 ) and high refractive index (typically $\mathrm{n} \simeq 2.5$ ), are meant to provide an uniform electric field penetration depth within a given spectral range but this aimed uniformity is never perfectly achieved. This then induces the additional phase-shift that should be accounted for. The way to minimize the effect of the additional phase-shift is to have the wavelength of the arc line be as close as possible to the wavelength used for the scanning of the science data cube. If we observe $\mathrm{H} \alpha$ close to its rest wavelength (which is the case for galactic HiI regions, inter-arm regions, planetary nebulae, SNR, or galaxies belonging to the Local Group for which systemic heliocentric radial velocities are low or even negative), the ideal situation would be to use a Hydrogen lamp to select the $\mathrm{H} \alpha$ emission as the calibration line, but in practice this is not a good option. The $\mathrm{H} \alpha$ emission line is intrinsically quite broad and it is then better to choose a narrower emission line close to the wavelength of $\mathrm{H} \alpha$ redshifted to the velocity of the target. As explained previously, the Ne $6598.95 \AA$ line (if a Neon lamp is available) can be successfully used as a reference line, given its intrinsic narrow shape and high intensity, allowing accurate measurements. For redshifted objects like nearby galaxies, this Ne line is very well suited because it is close to the observed redshifted $\mathrm{H} \alpha$ line $(\lambda=6598.95 \AA$ corresponds to the wavelength of $\mathrm{H} \alpha$ for an object with a radial velocity of $\sim 1650 \mathrm{~km} \mathrm{~s}^{-1}$ ). We note that, in order to take observations with a low-interference order Fabry-Perot, usually called a tunable filter, several lines in one calibration spectrum are needed to cover the broad FSR but for a high-order Fabry-Perot instrument, which has a small FSR, one calibration spectrum with one spectral line may be enough (if a second calibration spectrum is available one can then check the order). 


\section{B2 Interference filters}

\section{B2.1 Fabry-Perot transmission function}

In order to complement the basic Fabry-Perot formulae (1, 2, 3 and 4) given in section 2.2.1, we must introduce the Airy function. A Fabry-Perot provides a periodic signal described by the Airy function:

$\mathscr{A}(\phi)=\frac{1}{1+(2 \mathrm{~F} / \pi)^{2} \sin ^{2}(\phi / 2)}$

where $\phi$ is the total phase-shift (or phase-lag):

$\phi=\frac{2 \pi}{\lambda} \delta-2 \psi(\lambda)$

$\phi$ results from the combination of the optical travel path $\delta$ :

$\delta=2$ ne $\cos$ i

and $\psi(\lambda)$, the additional phase-lag described in section B1; $\mathrm{F}$ is the effective finesse; $\mathrm{n}$ is the refractive index of the medium between the two plates; $i$ is the beam inclination and $\mathrm{e}$ is the plate separation. The transmitted intensity $I_{t}$ of the Fabry-Perot is thus:

$\mathrm{I}_{\mathrm{t}}=\mathrm{I}_{\mathrm{t}}^{\max *} \frac{\mathrm{T}_{\mathrm{o}}^{2}}{\left(\mathrm{~A}_{\mathrm{o}}+\mathrm{T}_{\mathrm{o}}\right)^{2}} \mathscr{A}(\phi)$

with

$\mathrm{A}_{\mathrm{O}}+\mathrm{R}_{\mathrm{O}}+\mathrm{T}_{\mathrm{O}}=1$

where $I_{t}^{\max *}$ is the maximum transmitted intensity; A, R and $\mathrm{T}$, the absorption, reflectivity and transmissivity coefficients respectively. Note that $I_{t}=I_{t}^{\max *}$ when $\phi=2 p \pi$, which corresponds to the ideal case where absorption $\mathrm{A}_{\mathrm{o}}$ (and diffusion) could be neglected in the interfaces; in this case, formula B3 can be written as formula B1. Note also that the additional phase-lag can be as large as the main phase-lag $2 \pi \delta / \lambda$ when e and p are small, i.e. for a low-order Fabry-Perot, also called tunable filter.

\section{B2.2 Suitable interference filters}

Considering formulae B2, B3, B4 and B5, and as mentioned in Section 2.2.1, in order to select one or few orders, an interference filter must be chosen both for the target observation and for the calibration. In the case of the target observation, the central wavelength of the interference filter should correspond approximately to the mean velocity of the source. Depending on the science case, the observer can choose a filter allowing to select more than one FSR and make aposteriori correction but, in practice, more than three FSR may result in great confusion of orders, especially for galactic sources. In addition, all the continuum emission selected by the interference filter passes through the Fabry-Perot and it is modulated by the transmission curve of the filter. Thus, in order to increase the contrast of the Fabry-Perot (i.e. the monochromatic detection power), the interference filter should be as narrow as possible so that the continuum flux passing through the Fabry-Perot is minimised, which is an important point given that the photon noise $\sqrt{\mathrm{N}}$ of a continuum emission will limit the monochromatic detection. Moreover, in order to lower the continuum modulation, the passband of the filter should be as square as possible.

\section{B2.3 More on interference filters}

The observer has to take into account that the filter passband is blueshifted when (i) the incidence of the incoming light is not normal to the filter, (ii) the temperature drops and (iii) the filter ages. In addition, often under these circumstances the filter peak transmission is reduced and the FWHM is enlarged.

For small angles, the peak wavelength of the interference filter is a function of the beam aperture, temperature and time and it can be described by:

$\lambda_{\mathrm{c}} \simeq \lambda_{\text {beam }}-\alpha\left(\lambda_{\mathrm{o}}\right)\left(\mathrm{T}_{\mathrm{o}}-\mathrm{T}\right)-\mathrm{A}(\mathrm{t})$

where,

$\lambda_{\text {beam }} \simeq \lambda_{\mathrm{o}} \sqrt{1-\left(\frac{\mathrm{n}_{\mathrm{o}}}{\mathrm{n}_{\mathrm{f}}}\right)^{2} \sin ^{2}(\theta+\mathrm{i})}$

Here, $\lambda_{c}$ is the corrected central wavelength of the interference filter and $\lambda_{o}$ the central wavelength at normal incidence; $n_{o} \simeq 1$ and $n_{f}$ are the air refractive index and filter effective refractive index respectively; $\theta$ is the beam aperture (e.g. the focal ratio at SAM focus is $f / 16.63$ providing $\theta \simeq 1.7^{\circ}$ ) and $\mathrm{i}$ is the filter inclination; $T$ and $T_{O}$ are the ambient and reference temperature respectively, $\alpha\left(\lambda_{o}\right)$ a coefficient that slightly depends on the wavelength and finally $A(t)$ a coefficient that depends on the ageing of the filter and must be measured over a long enough period.

The three cases (i), (ii) and (iii) mentioned above will be discussed in the following.

(i) Narrowband interference filters are thin and solid Fabry-Perot interferometers usually operating in first order. The air gap is replaced by a thin layer of dielectric material (also called cavity) designed with an optical thickness optimized for the desirable transmission wavelength. Multiplecavity filters provide steeper band slopes (thus square passbands) by alternating layers of high-index material (typically $\mathrm{Ta}_{2} 0_{5}, \mathrm{n}_{\mathrm{H}} \simeq 2.13$ ) and low-index material (typically $\mathrm{SiO}_{2}$, $\mathrm{n}_{\mathrm{L}} \simeq 1.46$ ). The effective refractive index of an interference filter is typically $\mathrm{n}_{\mathrm{f}} \simeq \sqrt{\mathrm{n}_{\mathrm{H}} \mathrm{n}_{\mathrm{L}}} \simeq 1.76$.

We have measured in the lab at the Laboratoire d'Astrophysique de Marseille the central wavelength blueshift when inclining to a small angle $0^{\circ}<\mathrm{i}<6^{\circ}$ a set of $\sim 20$ interference filters. In order to experimentally measure $\mathrm{n}_{\mathrm{f}}$, but specifically to determine an empirical and operational relation, we fit equation (B8) to the data. We find $\mathrm{n}_{\mathrm{f}}=1.72_{-0.22}^{+0.35}$. The values vary quite a lot from one filter to another despite the fact that the process of fabrication is the same for all filters. For convenience, in table B1, we tabulate values computed for the blueshift $\left(\lambda_{c}-\lambda_{o}\right)$ using formula B8, in two cases, for $\lambda_{o}=\mathrm{H} \alpha 6562.78 \AA$ and $\lambda_{o}=\mathrm{H} \beta 4861 \AA$, using $\mathrm{n}_{\mathrm{f}}=1.72$.

In practice, the filter could be inclined to voluntarily shift the transmission curve to the blue, but the inclination should be small (typically lower than a couple of degrees) because it enlarges the passband and decreases the transmission. Moreover the shifts in wavelength do not increase linearly, becoming quite large beyond angles of 6 degrees.

In formula B8, the angle $\theta$ represents the aperture beam of the focal plane in which the filters are usually located. Usually Fabry-Perots are located in focal reducers to lower the focal ratio and increase the field of view. The aperture 
Table B1. Wavelength blueshift of interference filter transmission curves

\begin{tabular}{|c|c|c|}
\hline \multirow[t]{2}{*}{ inclination $\left({ }^{o}\right)$} & \multicolumn{2}{|c|}{ blue shift $(\AA)$} \\
\hline & $\mathrm{H} \beta 4861(\AA)$ & $\mathrm{H} \alpha 6563(\AA)$ \\
\hline 0 & 0 & 0 \\
\hline 1 & -0.2 & -0.3 \\
\hline 2 & -1.0 & -1.3 \\
\hline 3 & -2.1 & -3.0 \\
\hline 4 & -3.8 & -5.2 \\
\hline 5 & -6.0 & -8.1 \\
\hline 6 & -8.5 & -11.6 \\
\hline
\end{tabular}

$\theta$ is furthermore a telescope or focal reducer-dependent parameter. In the case of SAMI, given that it is not a focal reducer, the focal ratio in the filter plane is the one of the telescope (f/16.63).

(ii) The ambient and reference temperature are usually different. Manufacturers typically provide a reference temperature $\mathrm{T}_{\mathrm{o}}=20^{\circ} \mathrm{C}$ but the temperature when acquiring the observations is usually lower. To measure the temperature dependence, we used data provided by the company Melles Griot, Optics \& Photonics Company. From their data we obtained:

$$
\alpha\left(\mathrm{H}_{\alpha} 6562.78\right)=\frac{\Delta \lambda}{\Delta \mathrm{T}}=0.24 \pm 0.01 \mathrm{~K}^{-1}
$$

Other manufacturers provide lower values of $\alpha$, ranging from 0.15 to $0.19 K^{-1}$ in the R-band. Using the Melles Griot data, within the optical range, we computed a weak dependence of $\alpha$ with $\lambda$,

$$
\alpha=0.175\left(\frac{\lambda}{\lambda_{\mathrm{H} \alpha}}\right)+0.063,
$$

providing for instance $\alpha\left(\mathrm{H}_{\beta} 4861\right)=0.19 \pm 0.01 \mathrm{~K}^{-1}$.

(iii) The ageing effect on the wavelength (blueshift of the central wavelength) is very difficult to generalize because it affects differently each filter, even for a set of filters having the same age and built by the same company. It depends on the use and storage conditions. The filters measured in this experiment have, however, the same "history" and we measure $\mathrm{A}(\mathrm{t} \simeq 15$ years $) \simeq 3 \pm 3 \AA$. Some filters even displayed a red-shifted passband but measurements done 15 years apart may have been realised with different setups.

Moreover, transmission curves of old filters may completely collapse and their FWHM may dramatically increase. Peak transmissions and FWHMs of all filters that were 15 year-old were measured. In the worst cases, we find peak transmissions of $24-40 \%$ and as broad as $31-45 \AA$ (for filters showing, when new, a peak transmission of $70 \%$ and $\mathrm{FWHM}=20-24 \AA$ respectively). For the whole sample, we measured a mean peak transmission of $54 \pm 16 \%$ and a mean FWHM of $24 \pm 9.7 \AA$ to be compared with values, when new, of mean peak transmission of $71 \pm 2 \%$ and mean FWHM of $17.6 \pm 4.2 \AA$ (the FWHM scatter when the filters were new only reflects the fact that their initial widths ranged from 12 to $24 \AA$, but the increase on the scatter is significant). Here again, even if their process of fabrication, conditions of use and storage are similar, the scatter in peak transmissions and widths is very high. This means that some filters still exhibit good transmission curve even if they are 15 years old, while others that are just as old have low transmissions and they have been degraded with time. Thus, some low-transmission filters are clearly, in practice, not useful any longer. The conclusion is that filters must be regularly measured.

\section{B3 Determining FP parameters}

\section{B3.1 Determining the interference order $p$}

Formula (1) gives the interference order p. Although a number for the interference order is given by the manufacturer of the Fabry-Perot, it is also possible to measure it more accurately taking into account that the distance between the plates may vary slightly. This is, however, quite tricky, given that two different methods are needed to measure the integer part of $\mathrm{p}, \mathrm{p}_{\mathrm{o}}=\operatorname{int}(\mathrm{p})$, and its fractional residual, $\epsilon=\mathrm{p}-\operatorname{int}\left(\mathrm{p}_{\mathrm{o}}\right)$. However, for our purposes, the value of $\mathrm{p}_{\mathrm{o}}$ is the most important, which simplifies the computation. $\mathrm{p}_{\mathrm{o}}$ can be computed using a filter and a lamp whose spectrum has two emission lines in the wavelength range of interest. $\mathrm{p}_{\mathrm{o}}$ can be obtained using these two lines (two different wavelengths) using the classical method of coincidences. One can find the interference order for $\mathrm{p}_{0}$ by determining where in the field the interference rings coming from the two different wavelengths spatially coincide. Once this integer is known $\epsilon$ is computed by measuring the radius distribution of the parabolic rings.

\section{B3.2 Measuring the FSR}

In this section we explain how to measure the FSR for a Fabry-Perot in wavelength and in the so called binary control value (BCV, explained below), which is linked to the Fabry-Perot controller. These procedures can then be generalised for almost all scanning Fabry Perots in use.

Formulas 2 and 3 give the FSR in wavelength and as a function of the Fabry Perot plate separation, respectively. In this section we will detail how to compute and measure it. It is important to measure the FSR before a given observing run (from calibration cubes) in order to minimize the observing time spent on-object without losing any information. The goal is to do a scanning cycle on-object that corresponds exactly to one FSR or it is just slightly larger. If the scanning sequence covers less than one FSR, some areas of the field-of-view will not be observed and some wavelengths will be missing as well. If the scanning sequence covers $\mathrm{x} x$ FSR (with $\mathrm{x}>1$ ), some $(\mathrm{x}-1)$ interferograms are redundant and are useless because the new incomplete cycle does not cover a whole FSR. The FSR has to be measured at the calibration wavelength $\lambda_{c}$. If $\lambda_{c}$ is close to the observed wavelength $\lambda_{o}$, the additional phase-shift is negligible and, using equations (1) and (3), it is straightforward to compute the FSR at $\lambda_{o}$ from the FSR computed at $\lambda_{c}$. In practice, the determination of the FSR is done by scanning a gap slightly larger than the expected $\Delta e_{F S R}$ in order to get more than two maxima of the cyclic Airy function.

If $\mathrm{e}_{\mathrm{m}}$ is the mean distance between the plates around which the plates could be adjusted, and $\Delta e_{M}$ the maximum 
separation physically reachable between the plates (limited by the course of the piezoelectric actuators), the distance between the plates of the interferometer varies in the range $\sim\left[\left(\mathrm{e}_{\mathrm{m}}-\Delta \mathrm{e}_{\mathrm{M}}\right) / 2,\left(\mathrm{e}_{\mathrm{m}}+\Delta \mathrm{e}_{\mathrm{M}}\right) / 2\right]$. If one wants to control the parallelism between the two Fabry-Perot plates and move one plate with respect to the other, three degrees of freedom are needed: $X$ and $Y$ control the parallelism between the Fabry-Perot plates and $Z$ controls the spacing within a certain dynamic range. In our specific case, when using the CS 100 controller with the ICOS ET-65, the $Z$ offset between the plates has a full dynamic range of 12-bit binary number (note that for other Fabry Perot controllers, this dynamic range could be different - but this specific case is given here as an example). Thus, the maximum number of Binary Control Values (BCV) is $\Delta \mathrm{Z}_{\mathrm{M}}=2^{12}=4096$, spanning within the range $\left[-Z_{\mathrm{m}}=-2048, \mathrm{Z}_{\mathrm{m}}-1=+2047\right]$. $\Delta \mathrm{Z}_{\mathrm{M}}$ corresponds to approximately $2 \mu \mathrm{m}$ of plate separation adjustment. For the CS100 controller that drives the ICOS ET-65 etalon, the range of $\Delta \mathrm{e}_{\mathrm{M}} / 2 \simeq \pm 1 \mu \mathrm{m}$ around $\mathrm{e}_{\mathrm{m}}$ can be reached using a $\pm 10 \mathrm{~V}$ differential input, with a non-linearity of the scan of $\pm 1 \%$ and with a response time of $1 \mathrm{~ms}$ at a frequency response of $160 \mathrm{~Hz}(3 \mathrm{~dB})^{6}$. The smallest increment of plate separation adjustment $\delta e$ is thus

$\delta \mathrm{e}=\frac{\Delta \mathrm{e}_{\mathrm{M}}}{\Delta \mathrm{Z}_{\mathrm{M}}}$

Furthermore, the number of BCV needed to scan the full FSR, $\Delta \mathrm{Z}_{\mathrm{FSR}}$, is linked to the corresponding increment of plate separation to scan the FSR, $\Delta \mathrm{e}_{\mathrm{FSR}}$, and to the smallest increment of plate separation, $\delta \mathrm{e}$, by:

$\delta \mathrm{e}=\frac{\Delta \mathrm{e}_{\mathrm{FSR}}}{\Delta \mathrm{Z}_{\mathrm{FSR}}}$

$\delta \mathrm{e}$ is the physical expansion of the piezo-stacks for 1 BCV step applied to the etalon.

The exact value of the plate separation e and $\delta$ e are not known with high accuracy. We need to determine them using e.g. the calibration line $\lambda_{c}$. In order to switch from the expression of the FSR in terms of plate separation to the equation of the FSR as a function of the wavelength, we use equation (3) which gives $\Delta \mathrm{e}_{\mathrm{FSR}}=\lambda / 2$ at the centre of the rings $(\theta=0)$ for $\mathrm{n}=1$. Substituting equation B10 in the previous expression, we get $2 \delta \mathrm{e}=\lambda / \Delta \mathrm{Z}_{\mathrm{FSR}}$, which provides the definition for the "Fabry-Perot scanning constant" Q:

$\mathrm{Q}=2 \delta \mathrm{e}=\frac{\lambda}{\Delta \mathrm{Z}_{\mathrm{FSR}}}$

The "Fabry-Perot scanning constant" $\mathrm{Q}$ is equal to twice the smallest increment of plate separation $\delta$ e, conventionally given in units of $\AA / \mathrm{BCV}$. In the present case, for the ICOS ET-65 Fabry-Perot, equation (B9) provides a rough value $\mathrm{Q}_{\text {rough }} \simeq 2 \times 1 \mu \mathrm{m} / 2048 \simeq 9.8 \AA$ /BCV. The physical expansion of the piezo-stacks for $1 \mathrm{BCV}$ step $\delta$ e (thus Q) depends on the electronics of the controller that commands and controls the piezoelectric. In addition, it varies slightly with temperature, pressure and humidity and should be measured with accuracy. Several methods could be used, the simplest is to start from a given interferogram (ring image) and increase the $\mathrm{BCV}$ with very small steps, i.e. high sampling,

6 from ICOS, CS100 Controller and ET Series II User's Guide. to cover more (e.g. 10-20\% more) than the expected FSR. A highly oversampled calibration cube, with spectral coverage of more than one FSR, should be obtained in day time, ahead of the first night of observation, for this purpose and for computing the effective finesse. The image for which the initial pattern is observed a second time indicates that a FSR has been scanned and $\Delta \mathrm{Z}=\Delta \mathrm{Z}_{\mathrm{FSR}}$. For instance, applying this procedure to the high resolution Fabry-Perot with $\mathrm{p} \simeq 609$ at $\mathrm{Ne} 6598.95 \AA$, we measure $\Delta \mathrm{Z}_{\mathrm{FSR}} \simeq 705 \mathrm{BCV}$ (which is $\sim 17 \%$ of the maximum range $\Delta \mathrm{Z}_{\mathrm{M}}$ ). This now allows an accurate determination of $\mathrm{Q}$ using the relation B11: $\mathrm{Q}_{\mathrm{acc}}=9.360 \pm 0.003 / \mathrm{BCV}$ at $6598.95 \AA$.

\section{B3.3 Measuring the effective finesse}

Formulae B2 and B5 provide the Airy function which depends on the finesse $\mathrm{F}$. This section explains the nature of the finesse and how to measure it. In textbooks on FabryPerots, the finesse $F$ only depends on the reflectivity $R_{0}$ of the coating and could be computed using the optical finesse $F_{0}$ which is the mirror reflectivity finesse:

$\mathrm{F}_{\mathrm{o}}=\frac{\pi \sqrt{\mathrm{R}_{\mathrm{o}}}}{1-\mathrm{R}_{\mathrm{o}}}$.

In reality, Fabry-Perot interferometers differ in several ways from the ideal case and this impacts the actual finesse, that is enlarged. This actual finesse is referred to as the effective finesse. As described in section $B 1$, the reflectivity $R_{0}$ and the transmissivity $\mathrm{T}_{\mathrm{o}}$ coefficients depend on the wavelength (similar to the phase-shift, upon reflection at the plate surfaces). Moreover, the coating and the plate substrates induce absorption (described by equation B6), which also depends on the wavelength and reduces the transmitted intensity. Indeed, when the absorption $\mathrm{A}_{\mathrm{o}}$ increases, following equation B5, the transmitted intensity decreases. In addition, real Fabry-Perot interferometer plates present a variety of defects, they show irregularities at their surface, they are not perfectly parallel or planar and the plate separation e may vary due to instability in the control command of the Fabry-Perot. Finally the effective finesse of the interferometer will depend on different factors if the Fabry-Perot is located in a collimated (parallel) or in a converging beam. The Fabry-Perot could be located in a pupil plane, in a focal plane or somewhere in between along the optical plane. If the Fabry-Perot is at (or close to) a pupil plane it is usually in a collimated beam and if the Fabry-Perot is anywhere else, and a fortiori at (or close to) a focal plane, it is in a converging beam. In the most general case, the finesse $\mathrm{F}$ is the combination of the optical finesse $\mathrm{F}_{\mathrm{O}}$ and of the socalled control-command finesse $F_{c}$, imaging finesse $F_{i}$, aperture finesse $F_{a}$, and defect finesse $F_{d}$. Assuming the finesse functions are close to Gaussians (which is an acceptable first order approximation), the effective finesse is then:

$\frac{1}{\mathrm{~F}^{2}}=\frac{1}{\mathrm{~F}_{\mathrm{o}}^{2}}+\frac{1}{\mathrm{~F}_{\mathrm{c}}^{2}}+\frac{1}{\mathrm{~F}_{\mathrm{i}}^{2}}+\frac{1}{\mathrm{~F}_{\mathrm{a}}^{2}}+\frac{1}{\mathrm{~F}_{\mathrm{d}}^{2}}$

Depending on the location of the Fabry-Perot device along the optical path, this equation takes different forms. Independent of its location, the optical finesse $\mathrm{F}_{\mathrm{O}}$ and the controlcommand finesse $F_{c}$ always play a contribution. $F_{c}$ is due to control-command inaccuracies due to electronic instabilities 
in the plate separation positioning, mainly due to the piezoelectric actuators but also due to the gain and the timeconstant adjustments. In other words, the controller provides a plate separation e with an associated dynamic instability error $\delta$ e that affects the finesse. If the Fabry-Perot is placed in a converging beam, the beam aperture induces a shift on the transmitted wavelength as well as a decrease of the finesse. These two effects depend on the position in the field. Usually, the wavelength shift is the most important effect. The aperture finesse $F_{a}$ decreases when the beam aperture $f / d$ decreases (where $f$ is the focal length and $d$ the diameter of the mirror). In addition, $\mathrm{F}_{\mathrm{a}}$ also decreases when the interference order $\mathrm{p}$ increases, i.e. when the resolution increases, but it does not depend on the wavelength for a given p. It should be noted that the peak transmission (given by the equation B5) decreases as the beam aperture decreases, the effect being more pronounced the higher the order. On the other hand, the imaging finesse $F_{i}$ and the defects $F_{d}$ do not play a role because random point to point variations in the phase-shift between the plates do not affect the finesse in a converging beam (while it does in a collimated beam).

If the Fabry-Perot is placed at a collimated beam, then $f / d$ and $F_{a}$ tend to infinity, $1 / \mathrm{F}_{\mathrm{a}}^{2}$ tends to zero and the beam intercepts the whole surface of the Fabry-Perot (limited by the pupil). However, the light rays are never strictly parallel, and this affects the image quality. However, even if the rays were parallel, the image quality is also dependent on the angular size of the seeing at the Fabry-Perot entrance. This is accounted for by the imaging finesse $F_{i}$ defined as:

$\mathrm{F}_{\mathrm{i}}=\frac{\lambda}{2 \mathrm{ne} \Delta \theta \sin \theta}$,

where $\theta$ is the beam incidence on the Fabry-Perot and $\Delta \theta$, the image quality, i.e. the angular size corresponding to the seeing disk on the Fabry-Perot entrance. $F_{i}$ depends on the illumination of the Fabry-Perot and, therefore, on the input beam diameter. Note that for a given image quality, the imaging finesse depends on the plate separation and on the field-of-view. Finally, the defect finesse $F_{d}$ represents an important contribution to the effective finesse when the FP is at a collimated beam. Three potential defects may be associated with Fabry-Perot plates and are potential contributors to the instrumental broadening function (Atherton et al. 1981): (1) the surface irregularities (roughness), coating deposit inhomogeneity on the plates; (2) the departures from parallelism ("wedging") between the plates and (3) the bowing of the plates due to mechanical stress because of the coating or the opto-mechanic interfaces. The defects mentioned in (2) and (3) also depend on gravity (i.e. on the position of the interferometer during telescope tracking). These three defects account respectively for the surface quality finesse $F_{d, s}$, the wedging finesse $F_{d, w}$ and the bowing finesse $F_{d, b}$. Finally the defect finesse could be written as:

$\frac{1}{\mathrm{~F}_{\mathrm{d}}^{2}}=\frac{1}{\mathrm{~F}_{\mathrm{d}, \mathrm{s}}^{2}}+\frac{1}{\mathrm{~F}_{\mathrm{d}, \mathrm{w}}^{2}}+\frac{1}{\mathrm{~F}_{\mathrm{d}, \mathrm{b}}^{2}}=\frac{32 \ln 2\left(\delta \mathrm{d}_{\mathrm{s}}\right)^{2}+3\left(\delta \mathrm{d}_{\mathrm{w}}\right)^{2}+2\left(\delta \mathrm{d}_{\mathrm{b}}\right)^{2}}{\lambda^{2}}$

where $\delta \mathrm{d}_{\mathrm{s}}$ is the gaussian standard deviation (r.m.s) of the polishing plus coating errors, $\delta \mathrm{d}_{\mathrm{w}}$ the maximum wedge amplitude (i.e. the peak-to-valley deviation from parallelism) and $\delta \mathrm{d}_{\mathrm{b}}$ the maximum (the peak-to-valley) ex- cursion from the plane surface (i.e. the sagitta of the arc segment). All may cause blurring (broadening) of the lineshapes. The slightest misalignment of the etalons' plates can lead to measurements of the effective finesse and resolution that are slightly different from one run to the next, given that the system setup is fine-tuned for each run.

In practice, the effective finesse, $\mathrm{F}$ can be easily measured during the setup. This is achieved by obtaining e.g. a scanning sequence at high sampling also needed for computation of the FSR. We use the formula:

$\mathrm{F}=\frac{\Delta \mathrm{e}_{\mathrm{FSR}}}{\Delta \mathrm{e}_{\mathrm{A}}}$

where $\Delta \mathrm{e}_{\mathrm{A}}$ is the FWHM of the Airy function or, more precisely, it is the increment in plate separation necessary to cover one FWHM of the Airy function while $e_{F S R}$ is the increment of plate separation necessary to scan the FSR.

Using equations (3) and (B16) we find:

$\Delta \mathrm{e}_{\mathrm{A}}=\frac{\mathrm{e}}{\mathrm{pF}}$.

The average effective finesse for the whole field-of-view can be obtained by fitting an Airy function to the arc lines of each calibration spectrum, in the data cube. In practice it is sufficient to extract a $1 \mathrm{D}$ spectrum from a calibration data cube, e.g. in the centre of its rings (where $\theta=0$ ) and to fit an Airy function or simply measure the FWHM of an arc line. $\mathrm{F}$ is then obtained by dividing FSR by the width of the arc line. For the high-resolution Fabry Perot used for this study we measure an effective finesse $\mathrm{F} \simeq 18.48$ at $\mathrm{Ne} 6598.95 \AA$ which gives $\mathrm{R} \simeq \mathrm{pF} \simeq 11260$ at $\mathrm{Ne} 6598.95 \AA$ and $\mathrm{R} \simeq 11200$ at $\mathrm{H} \alpha$.

\section{B3.4 Defining the scanning sequence}

This section describes how to define the scanning sequence. Once the number of channels $\mathrm{n}$ is fixed and the Fabry-Perot constant $\mathrm{Q}$ is known, the scanning sequence could be defined for a given wavelength. Reference wavelength calibration data cubes are taken at the calibration wavelength $\lambda_{c}$ (e.g. using the Ne I emission line $6598.95 \AA$ selected through a narrow interference filter $\sim 19 \AA$ ) by scanning the FSR at $\lambda_{c}$. A BCV value close to $\mathrm{Z}=0$ (the middle of the plate separation adjustment) defines the zero point of the scanning sequence that corresponds to half of the FSR to scan. Nevertheless, other values different from $\mathrm{Z}=0$ can also be chosen as long as the FSR fits within the range of $\mathrm{BCV}$ values (i.e., within $\pm \mathrm{Z}_{\mathrm{m}}$, e.g. at SAM-FP we regularly use $\mathrm{Z}=2048$ with the scanning sequence going from 0 to 4095). Using the equation B11, e.g. at the calibration wavelength $\lambda_{c}$, the first channel to scan thus corresponds to the BCV: $\mathrm{Z}_{1}=-\Delta \mathrm{Z}_{\mathrm{FSR}} / 2=-\lambda_{\mathrm{c}} / 2 \mathrm{Q}$; the scanning step between two successive channels is $\Delta Z_{\mathrm{FSR}} / \mathrm{n}=\lambda_{\mathrm{c}} / \mathrm{nQ}$, thus channel 2 corresponds to $Z_{2}=-\lambda_{c} / 2 Q+\lambda_{c} / n Q$, channel 3 to $Z_{3}=\lambda_{\mathrm{c}} / 2 \mathrm{Q}+2 \lambda_{\mathrm{c}} / \mathrm{nQ}$ and the last channel is $Z_{\mathrm{n}}=-\lambda_{\mathrm{c}} / 2 \mathrm{Q}+(\mathrm{n}-1) \lambda_{\mathrm{c}} / \mathrm{nQ}$. Ideally, we should plan to not have an overlapping of two successive orders, thus channel 1 and channel $n+1$ are identical. The first and the $(n+1)^{\mathrm{st}}$ channels match, as do also the second and the $(n+2)^{\text {nd }}$ and so on. Finally, the step in BCV values between two successive channels $\Delta \mathrm{Z}_{\mathrm{FSR}} / \mathrm{n}$ is an integer value, thus $\mathrm{n} \times \operatorname{nint}\left(\Delta \mathrm{Z}_{\mathrm{FSR}} / \mathrm{n}\right)$, where nint is the function nearest integer, is not exactly 
equal to $\Delta Z_{F S R}$. The difference $\left|\Delta Z_{\mathrm{FSR}}-\mathrm{n} \times \operatorname{nint}\left(\Delta \mathrm{Z}_{\mathrm{FSR}} / \mathrm{n}\right)\right|$ must then be homogeneously spread over all steps of the scanning sequence. This means that, in the end, the step between any two successive channels could be one BCV larger or smaller than the average step value. However, this is not a problem given that the FSR is, in any case, scanned with an accuracy better than one BCV (i.e. better than $0.14 \%$ for the setup used in this paper).

On-sky observations are carried out using the same scanning sequence (number of channels) as for the calibration but at the wavelength of the observation $\lambda_{o}$, in order to cover the whole FSR. The number of BCV scanned is then not exactly the same as for the calibration. Indeed, to cover a full FSR at the wavelength of the on-sky observation $\lambda_{o}$, a new sequence should be defined by:

$$
\left[-\frac{\lambda_{\mathrm{o}}}{2 \mathrm{Q}},-\frac{\lambda_{\mathrm{o}}}{2 \mathrm{Q}}+\frac{\lambda_{\mathrm{o}}}{\mathrm{nQ}},-\frac{\lambda_{\mathrm{o}}}{2 \mathrm{Q}}+\frac{2 \lambda_{\mathrm{o}}}{\mathrm{nQ}}, \ldots,-\frac{\lambda_{\mathrm{o}}}{2 \mathrm{Q}}+\frac{\mathrm{n}-1}{\mathrm{nQ}} \lambda_{\mathrm{o}}\right]
$$

The zero point of the scanning sequence $(Z=0)$ should be common to all wavelength, i.e. the plate separation e of the Fabry-Perot should be the same regardless the wavelength because the equation $\mathrm{p}_{\mathrm{c}} \lambda_{\mathrm{c}}=\mathrm{p}_{\mathrm{o}} \lambda_{\mathrm{o}}$, deduced from the equation (1) for each point in the field of view (for a fixed $\cos \theta$ ), is only valid for this plate separation e. Consequently, if $\lambda_{\mathrm{o}} \lessgtr \lambda_{\mathrm{c}}, \Delta \mathrm{Z}_{\mathrm{FSR}}\left(\lambda_{\mathrm{o}}\right) \lessgtr \Delta \mathrm{Z}_{\mathrm{FSR}}\left(\lambda_{\mathrm{c}}\right)$. Therefore, besides the additional phase-shift mentioned in the beginning of this appendix, another reason to choose $\lambda_{c}$ as close as possible to $\lambda_{o}$ is to minimize the difference between the two FSR, minimizing the need for interpolation during the construction of the wavelength calibrated data cube. Nevertheless, here again, the observer is free to use other scanning sequences, if desired. 